\title{
Encuesta M apCom 2017 a la comunidad de investigadores de la comunicación en España
}

\author{
J osé L uis Piñuel Raigada (U CM ) \\ pinuel@ucm.es \\ Carlos L ozano A scencio (URJC) \\ carlos.lozano@urjc.es \\ J uan A ntonio Gaitán M oya (U CM ) \\ jagamo@ucm.es \\ Carmen Caffarel Serra (URJC) \\ carmen.caffarel@urjc.es
}

\begin{abstract}
Resumen: Entre mayo y agosto de 2017, cerca de 2500 investigadores de la comunicación, censados como doctores y estudiantes de doctorado en centros universitarios españoles con titulaciones de grado y posgrado en las carreras de comunicación, fueron convocados por el Proyecto M apCom (CSO2013-47933-C4), en su tercera fase de desarrollo, a responder una encuesta en línea diseñada para conocer los perfiles de su identidad profesional y académica, así como la percepción sobre su estatus y su práctica investigadora.

A ccedieron al formulario de esta encuesta 1254 investigadores, de los cuales 838 (el $34 \%$ del censo) respondieron a las preguntas, 496 finalizaron el formulario y 342 lo respondieron parcialmente. Por último, 416 abandonaron sin contestar ninguna pregunta.

Las respuestas relacionadas con la identidad profesional y académica tienen que ver con la experiencia investigadora y sus reconocimientos: integración en grupos consolidados y en equipos de proyectos, sexenios, pertenencia a sociedades científicas, registro en bases de datos de investigación, etc. Las contestaciones relacionadas con la apreciación de su propia actividad investigadora destacan por denunciar mayoritariamente el escaso reconocimiento de la dedicación a investigar frente a la dedicación docente, cuando, de forma paradójica, es el resultado de la investigación del que se hace depender las evaluaciones de la carrera académica y de la calidad de las titulaciones que se imparten.
\end{abstract}

Palabras clave: metainvestigación en comunicación, encuesta, M apCom, universidad española. 
Abstract: A bout 2500 communication researchers, registered as doctors and doctoral students in Spanish university centres with undergraduate and postgraduate degrees in Communication, are invited from $M$ ay to $A$ ugust 2017 by the $M$ apCom Project (CSO2013-47933-C4) in its third phase of development to answer an online survey designed to know the profiles of their professional and academic identity, as well as the perception of their status and their research practice.

The survey form was accessed by 1,254 researchers, of whom 838 (34\% of the census) answered the questions, 496 completed the form and 342 partially answered. Finally, 416 left without answering any questions.

The answers related to the professional and academic identity have to do with the research experience and its acknowledgments: integration in consolidated groups and project teams, six-year terms, membership in scientific societies, registration in research databases, etc. The answers related to the appreciation of their own research activity mainly stand out for denouncing the scarce recognition to research dedication as opposed to teaching dedication, when, paradoxically, the eval uations of the academic career and the quality of the degrees taught depend on the academic career evaluations.

Keywords: M etaresearch in Communication, survey, M apCom, Spanish 


\section{CONTEXTUALIZACIÓN Y METODOLOGÍA}

En las fases previas del proyecto mencionado se desvelaron los ejes sobre los que giran los discursos científicos, tanto en los productos de investigación de mayor rango - tesis doctorales y proyectos competitivos I $+D$ de ámbito estatal (fase 1.a)- como en las discusiones habidas durante las sesiones cel ebradas en M adrid, Barcelona y $M$ álaga con grupos representativos de los procesos investigadores (fase 2.a). El Proyecto $M$ apCom finaliza en su tercera fase con la realización de una encuesta en línea aplicada al universo de investigadores del territorio español censados en registros académicos de las universidades con titulaciones de grado y posgrado en Comunicación, así como en sociedades científicas de este ámbito académico, al objeto de cartografiar los resultados para descubrir cómo se configuran las representaciones sociales de los investigadores académicos del ámbito en lo que se refiere a su propia actividad científica, y completar de este modo el objetivo de elaborar un mapa de proyectos, grupos, líneas, objetos de estudio y métodos de investigación sobre prácticas sociales de comunicación en España. Nuestra aspiración es que el Proyecto M apCom sirva de referencia a las entidades nacionales y autonómicas responsables de evaluar solicitudes e informes en las convocatorias de proyectos, y promover políticas científicas para la coordinación de la red de equipos de investigación académica.

\section{Diseño de la población encuestada y muestra}

Por lo que respecta al universo de destinatarios de la encuesta, se decidió tomar en consideración:

a) Investigadores censados en facultades de Comunicación en España (todos los doctores en nómina de cada universidad)

b) Investigadores miembros de sociedades científicas (miembros doctores que no estén en nómina en ninguna universidad)

c) Investigadores en formación en los programas de doctorado

El universo del primer grupo está conformado por todos los profesores doctores en las facultades con estudios de grado universitario en Comunicación, lo que comprende todas las universidades incluidas en el universo de tesis doctorales y proyectos $I+D$ de la primera fase de $\mathrm{M}$ apCom. El segundo grupo contempla a los doctores que no imparten docencia universitaria, pero que sí pertenecen a alguna sociedad científica, desde las 
cuales se facilitó el acceso a la encuesta. R especto al tercer grupo de investigadores en formación en los programas de doctorado, Ios equipos de las áreas M apCom contactaron con los coordinadores de los programas de doctorado para que utilizaran sus censos y enviaran a los doctorandos de último curso el enlace de la encuesta.

Tras elaborar el censo correspondiente a los universos citados, se cursaron un total de 2418 invitaciones personalizadas a investigadores adscritos a universidades españolas, así como una invitación abierta indeterminada a través de sociedades científicas y programas de doctorado. Se obtuvieron 838 contestaciones para una tasa de respuesta efectiva de $34,66 \%$. Para estimar la representatividad por estratos de la muestra obtenida, se tomaron en cuenta las variables de universidad (Tabla 1) y comunidad autónoma (Tabla 2) a las que pertenecía el investigador. Específicamente, se estimaron los porcentajes esperados mínimos y máximos para cada categoría de las variables, con el fin de comprobar que ninguna de las universidades o comunidades autónomas (CC. A A .) quedaran infra- 0 suprarrepresentadas en la muestra final con el sesgo de autorrespuesta. Para ello, se calculó en el censo la media y la desviación típica de cada subcategoría (utilizando la variable dummy para cada valor), y se obtuvieron así intervalos de confianza ( $\%$ mín = M -DT y \% máx = M +DT). Como se observa en las tablas, los porcentajes de cada universidad y CC. A A. encajaron dentro de los límites esperados, por lo que así los estratos en la muestra están correctamente representados con respecto a los del censo.

TABLA 1

Distribución de investigadores invitados y proporción de respuestas por comunidad autónoma y con porcentajes esperados mínimos y máximos para cada estrato

\begin{tabular}{lrrrrrr}
\hline \multicolumn{1}{c}{ CC.AA. } & Invitados & \% & M uestra & $\begin{array}{c}\text { \% } \\
\text { M uestra }\end{array}$ & $\begin{array}{r}\% \text { M ín. } \\
\text { esperado }\end{array}$ & $\begin{array}{r}\% \text { M áx. } \\
\text { esperado }\end{array}$ \\
\hline A ndalucía & 253 & 10,5 & 77 & $9,20 \%$ & $-20,15 \%$ & $41,07 \%$ \\
A ragón & 43 & 1,8 & 19 & $2,30 \%$ & $-11,44 \%$ & $15,00 \%$ \\
A sturias & 0 & 0 & 1 & $0,10 \%$ & $0,00 \%$ & $0,00 \%$ \\
Baleares & 0 & 0 & 7 & $0,80 \%$ & $0,00 \%$ & $0,00 \%$ \\
Canarias & 15 & 0,6 & 2 & $0,20 \%$ & $-7,23 \%$ & $8,47 \%$ \\
Cantabria & 0 & 0 & 0 & $0,00 \%$ & $0,00 \%$ & $0,00 \%$ \\
Castilla-La M ancha & 20 & 0,8 & 4 & $0,50 \%$ & $-8,23 \%$ & $9,89 \%$ \\
Castilla y León & 121 & 5 & 61 & $7,30 \%$ & $-16,81 \%$ & $26,81 \%$ \\
Cataluña & 405 & 16,7 & 94 & $11,20 \%$ & $-20,60 \%$ & $54,10 \%$ \\
Extremadura & 36 & 1,5 & 7 & $0,80 \%$ & $-10,62 \%$ & $13,60 \%$ \\
Galicia & 110 & 4,5 & 39 & $4,70 \%$ & $-16,29 \%$ & $25,39 \%$
\end{tabular}


TABLA 1

Distribución de investigadores invitados y proporción de respuestas por comunidad autónoma y con porcentajes esperados mínimos y máximos para cada estrato (cont.)

\begin{tabular}{lcrrrrr}
\hline \multicolumn{1}{c}{ CC.AA. } & Invitados & \% & M uestra & $\begin{array}{c}\text { \% } \\
\text { M uestra }\end{array}$ & $\begin{array}{c}\text { \% Mín. } \\
\text { esperado }\end{array}$ & $\begin{array}{r}\text { \% Máx. } \\
\text { esperado }\end{array}$ \\
\hline La Rioja & 26 & 1,1 & 13 & $1,60 \%$ & $-9,24 \%$ & $11,40 \%$ \\
M adrid & 770 & 31,8 & 146 & $17,40 \%$ & $-14,76 \%$ & $78,44 \%$ \\
M urcia & 84 & 3,5 & 32 & $3,80 \%$ & $-14,85 \%$ & $21,79 \%$ \\
Navarra & 55 & 2,3 & 30 & $3,60 \%$ & $-12,64 \%$ & $17,18 \%$ \\
País Vasco & 190 & 7,9 & 35 & $4,20 \%$ & $-19,05 \%$ & $34,77 \%$ \\
Valencia & 290 & 12 & 79 & $9,40 \%$ & $-20,51 \%$ & $44,49 \%$ \\
Fuera de España & & & 5 & $0,60 \%$ & & \\
Sin contestación & & & 187 & $22,30 \%$ & & \\
Total & $\mathbf{2 4 1 8}$ & $\mathbf{1 0 0}$ & $\mathbf{8 3 8}$ & $\mathbf{1 0 0 , 0 0 \%}$ & & \\
\hline
\end{tabular}

TABLA 2

Distribución de investigadores invitados y proporción de respuestas por universidad con porcentajes esperados mínimos y máximos para cada estrato (cont.)

\begin{tabular}{lcccccc}
\hline \multicolumn{1}{c}{ Universidad } & Invitados & $\%$ & M uestra & $\begin{array}{c}\% \\
\text { Muestra }\end{array}$ & $\begin{array}{c}\text { \% Mín. } \\
\text { esperado }\end{array}$ & $\begin{array}{r}\% \text { Máx. } \\
\text { esperado }\end{array}$ \\
\hline CEU SA PA BL & 126 & 5,2 & 8 & $1,00 \%$ & $31,84 \%$ & $27,44 \%$ \\
EUCCB & 1 & 0 & 0 & $0,00 \%$ & $-1,99 \%$ & $2,07 \%$ \\
MU & 14 & 0,6 & 1 & $0,10 \%$ & $-7,01 \%$ & $8,17 \%$ \\
Nebrija & 43 & 1,8 & 0 & $0,00 \%$ & $-11,44 \%$ & $15,00 \%$ \\
UA & 62 & 2,6 & 11 & $1,30 \%$ & $-13,25 \%$ & $18,37 \%$ \\
UAB & 98 & 4,1 & 12 & $1,40 \%$ & $-15,67 \%$ & $23,77 \%$ \\
UBU (Burgos) & 12 & 0,5 & 2 & $0,20 \%$ & $-6,53 \%$ & $7,53 \%$ \\
UC3M & 84 & 3,5 & 13 & $1,60 \%$ & $-14,85 \%$ & $21,79 \%$ \\
UCA & 31 & 1,3 & 3 & $0,40 \%$ & $-9,97 \%$ & $12,53 \%$ \\
UCAM & 23 & 1 & 4 & $0,50 \%$ & $-8,76 \%$ & $10,66 \%$ \\
UCH-CEU & 32 & 1,3 & 2 & $0,20 \%$ & $-10,11 \%$ & $12,75 \%$ \\
UCJC & 35 & 1,4 & 1 & $0,10 \%$ & $-10,50 \%$ & $13,40 \%$ \\
UCLM & 20 & 0,8 & 4 & $0,50 \%$ & $-8,23 \%$ & $9,89 \%$ \\
UCM & 307 & 12,7 & 55 & $6,60 \%$ & $-20,60 \%$ & $46,00 \%$ \\
UD & 32 & 1,3 & 5 & $0,60 \%$ & $-10,11 \%$ & $12,75 \%$
\end{tabular}


TABLA 2

Distribución de investigadores invitados y proporción de respuestas por universidad con porcentajes esperados mínimos y máximos para cada estrato (cont.)

\begin{tabular}{|c|c|c|c|c|c|c|}
\hline Universidad & Invitados & $\%$ & M uestra & $\begin{array}{c}\% \\
\text { M uestra }\end{array}$ & $\begin{array}{l}\text { \% M ín. } \\
\text { esperado }\end{array}$ & $\begin{array}{l}\text { \% Máx. } \\
\text { esperado }\end{array}$ \\
\hline UDC & 30 & 1,2 & 10 & $1,20 \%$ & $-9,83 \%$ & $12,31 \%$ \\
\hline$U d G$ & 49 & 2 & 3 & $0,40 \%$ & $-12,06 \%$ & $16,12 \%$ \\
\hline UDIMA & 35 & 1,4 & 7 & $0,80 \%$ & $-10,50 \%$ & $13,40 \%$ \\
\hline $\mathrm{UdL}$ & 13 & 0,5 & 2 & $0,20 \%$ & $-6,77 \%$ & $7,85 \%$ \\
\hline UEMC (Valladolid) & 9 & 0,4 & 2 & $0,20 \%$ & $-5,72 \%$ & $6,46 \%$ \\
\hline UGR & 42 & 1,7 & 13 & $1,60 \%$ & $-11,33 \%$ & $14,81 \%$ \\
\hline $\begin{array}{l}\text { UIB-CESAG (P. } \\
\text { Comillas) }\end{array}$ & 23 & 1 & 6 & $0,70 \%$ & $-8,76 \%$ & $10,66 \%$ \\
\hline UIC & 16 & 0,7 & 6 & $0,70 \%$ & $-7,45 \%$ & $8,77 \%$ \\
\hline UJ। & 55 & 2,3 & 28 & $3,30 \%$ & $-12,64 \%$ & $17,18 \%$ \\
\hline ULL & 15 & 0,6 & 2 & $0,20 \%$ & $-7,23 \%$ & $8,47 \%$ \\
\hline UM & 61 & 2,5 & 28 & $3,30 \%$ & $-13,08 \%$ & $18,04 \%$ \\
\hline UMA & 86 & 3,6 & 29 & $3,50 \%$ & $-14,97 \%$ & $22,09 \%$ \\
\hline UMH & 54 & 2,2 & 8 & $1,00 \%$ & $-12,55 \%$ & $17,01 \%$ \\
\hline UNAV & 55 & 2,3 & 29 & $3,50 \%$ & $-12,64 \%$ & $17,18 \%$ \\
\hline UNED & 1 & 0 & 4 & $0,50 \%$ & $-1,99 \%$ & $2,07 \%$ \\
\hline UNEX & 35 & 1,4 & 6 & $0,70 \%$ & $-10,50 \%$ & $13,40 \%$ \\
\hline UNIR & 26 & 1,1 & 13 & $1,60 \%$ & $-9,24 \%$ & $11,40 \%$ \\
\hline UNIZAR & 43 & 1,8 & 18 & $2,10 \%$ & $-11,44 \%$ & $15,00 \%$ \\
\hline UOC & 15 & 0,6 & 10 & $1,20 \%$ & $-7,23 \%$ & $8,47 \%$ \\
\hline UPF & 73 & 3 & 24 & $2,90 \%$ & $-14,10 \%$ & $20,14 \%$ \\
\hline UPSA & 31 & 1,3 & 10 & $1,20 \%$ & $-9,97 \%$ & $12,53 \%$ \\
\hline UPV & 26 & 1,1 & 12 & $1,40 \%$ & $-9,24 \%$ & $11,40 \%$ \\
\hline UPV /EHU & 143 & 5,9 & 29 & $3,50 \%$ & $-17,68 \%$ & $29,50 \%$ \\
\hline URJC & 117 & 4,8 & 52 & $6,20 \%$ & $-16,62 \%$ & $26,30 \%$ \\
\hline URL & 80 & 3,3 & 21 & $2,50 \%$ & $-14,58 \%$ & $21,20 \%$ \\
\hline URV & 18 & 0,7 & 7 & $0,80 \%$ & $-7,86 \%$ & $9,34 \%$ \\
\hline
\end{tabular}


TABLA 2

Distribución de investigadores invitados y proporción de respuestas por universidad con porcentajes esperados mínimos y máximos para cada estrato (cont.)

\begin{tabular}{lcccccc}
\hline \multicolumn{1}{c}{ Universidad } & Invitados & $\%$ & M uestra & $\begin{array}{c}\% \\
\text { M uestra }\end{array}$ & $\begin{array}{c}\% \text { Mín. } \\
\text { esperado }\end{array}$ & $\begin{array}{r}\% \text { Máx. } \\
\text { esperado }\end{array}$ \\
\hline US & 94 & 3,9 & 26 & $3,10 \%$ & $-15,44 \%$ & $23,22 \%$ \\
USA & 19 & 0,8 & 16 & $1,90 \%$ & $-8,04 \%$ & $9,62 \%$ \\
USC & 49 & 2 & 20 & $2,40 \%$ & $-12,06 \%$ & $16,12 \%$ \\
UV & 61 & 2,5 & 18 & $2,10 \%$ & $-13,17 \%$ & $18,21 \%$ \\
UVA (Segovia) & 29 & 1,2 & 0 & $0,00 \%$ & $-9,69 \%$ & $12,09 \%$ \\
UVA (Valladolid) & 21 & 0,9 & 30 & $3,60 \%$ & $-8,41 \%$ & $10,15 \%$ \\
UVIC & 43 & 1,8 & 7 & $0,80 \%$ & $-11,44 \%$ & $15,00 \%$ \\
Uvigo & 31 & 1,3 & 9 & $1,10 \%$ & $-9,97 \%$ & $12,53 \%$ \\
Otros casos (foráneas) & & & 19 & $2,00 \%$ & & \\
Sin contestar & & 193 & $23,00 \%$ & & \\
Total & $\mathbf{2 4 1 8}$ & $\mathbf{1 0 0}$ & $\mathbf{8 3 8}$ & $\mathbf{9 9 , 7 0 \%}$ & & \\
\hline
\end{tabular}

En resumen, de los 2418 invitados a contestar la encuesta mediante un correo electrónico personalizado y la entrega de una contraseña para abrir el formulario, accedieron al cuestionario 1254 investigadores, de los cuales 838 (el $34 \%$ del censo) respondieron a las preguntas, 496 finalizaron el formulario y 342 lo respondieron parcialmente. Por último, 416 abandonaron sin contestar ninguna pregunta.

\section{EI formulario de encuesta}

Con el propósito de conocer los perfiles de la identidad académica de los investigadores y las valoraciones de su experiencia en la investigación desarrollada en el campo de la comunicación, el cuestionario (A nexo 1) comprendió los apartados generales siguientes:

- Información previa del encuestado sobre el Proyecto M apCom, al objeto de estimar si el interés mostrado al contestar la encuesta se podría deber al conocimiento previo sobre este proyecto. 
- Perfil como investigador (antigüedad, proyectos realizados, registros en bases de datos, sexenios, etc.) para poder relacionarlo con las respuestas que posteriormente se ofrecían al ser preguntado sobre su experiencia investigadora.

- Adscripción profesional como investigador para relacionar su perfil de investigador con los datos del censo de investigadores (universidad de adscripción, rango académico/laboral, grupos/equipos de investigación, comunidad autónoma, docencia ejercida, etc.).

- Experiencia investigadora, para recabar información referida a la experiencia personal en la actividad investigadora desarrollada, tomando en consideración la fijación de objetivos, la elección de objetos de estudio, el tipo de técnicas empleadas en la elaboración y registro de datos y la valoración de esta experiencia referida al beneficio obtenido como retorno.

- Debilidades y fortalezas de la investigación en comunicación, al valorar negativa o positivamente las condiciones materiales, organizacionales e institucionales a las que, según su experiencia, ha estado sometida en España la actividad investigadora.

- Inserción en los perfiles sociodemográficos del censo de investigadores en comunicación al responder a las preguntas sobre edad, género, formación de origen y rango de ingresos económicos por la actividad profesional.

Con los datos obtenidos de esta encuesta, aspiramos a elaborar su cartografía territorial por comunidades autónomas y universidades, al objeto de poder situar geográficamente el estado y desarrollo de la actividad investigadora en comunicación que la universidad española realiza ya sea compitiendo, ya sea complementando su actividad docente. Pero, sobre todo, para poder ofrecer estos datos a libre disposición de la comunidad científica, poniéndolos además en relación con los datos ya cartografiados territorialmente y referidos a la producción de las investigaciones de mayor rango, como son las tesis doctorales y los proyectos I+D de convocatorias estatales.

\section{RESULTADOSY DISCUSIÓN}

L a participación constatada al comprobar los estratos de la muestra por universidades y CC. A A respecto al censo nos permite interpretar los datos obtenidos sabiendo que estos son representativos del universo de investigadores de la comunicación en la universidad española con titulaciones en Comunicación. Veamos a continuación los perfiles más destacados en cada uno de los apartados de la encuesta. 


\section{Información previa del encuestado sobre el Proyecto M apC om}

El acceso y la cumplimentación del formulario de encuesta muestran perfiles diferenciales en función del conocimiento previo del Proyecto $\mathrm{M}$ apCom que manifiesta el encuestado. En este sentido, destaca el dato de que solo 9 encuestados (1,1\%) dejaron sin contestar la pregunta inicial y que, contestada esta eligiendo al guna de las respuestas al ternativas, un $67 \%$ manifestó que no lo conocía o solo de nombre, y un $22 \%$ que conocía M apCom por otras vías, que para el $10 \%$ restante consistieron en consultar la web y sus bases de datos, es decir, que disponían de conocimiento exhaustivo 0 al menos de conocimiento parcial.

Por CC. A A . y universidades destaca la Comunidad Valenciana con un $24,1 \%$ de encuestados cuyo conocimiento es, o bien exhaustivo, 0 al menos parcial de las bases de datos y publicaciones de $\mathrm{M}$ apCom, y dentro de esta comunidad, la UJI destaca porque el $7 \%$ de los encuestados de esta universidad manifestó conocer exhaustiva o parcialmente el trabajo $\mathrm{M}$ apCom. L uego siguen M adrid y Cataluña con un $17 \%$ y un $10,7 \%$ respectivamente de encuestados que manifiestan un conocimiento exhaustivo 0 al menos parcial de las bases de datos de M apCom, y dentro de ellas, la URL, que destaca con un $6,4 \%$ de los encuestados de esta universidad cuyo conocimiento es exhaustivo 0 al menos parcial de $\mathrm{M}$ apCom.

En conjunto, contemplando los datos anteriores y a la luz de quienes manifiestan su conocimiento previo de $\mathrm{M}$ apCom con relación al uso o no de la web del proyecto y con el nivel de participación en sus trabajos, obtenemos los datos que se muestran en la Tabla 3:

TABLA 3

Usuario web y nivel de participación en el Proyecto M A PCOM

\begin{tabular}{lccccc}
\hline & $\begin{array}{c}\text { He participado } \\
\text { en los } \\
\text { equipos por } \\
\text { áreas }\end{array}$ & $\begin{array}{c}\text { He sido } \\
\text { invitado a } \\
\text { participar o } \\
\text { me han } \\
\text { consultado en } \\
\text { la recopilación } \\
\text { de datos }\end{array}$ & $\begin{array}{c}\text { No he } \\
\text { participado }\end{array}$ & $\begin{array}{c}\text { Sin } \\
\text { contestación }\end{array}$ & Total \\
\hline No usuario & 37 & 154 & 555 & & \\
web & $4,9 \%$ & $20,6 \%$ & $74,2 \%$ & $0,3 \%$ & $100,0 \%$ \\
& $63,8 \%$ & $86,0 \%$ & $93,8 \%$ & $22,2 \%$ & $89,3 \%$ \\
& $4,4 \%$ & $18,4 \%$ & $66,2 \%$ & $0,2 \%$ & $89,3 \%$ \\
\hline
\end{tabular}


TABLA 3

Usuario web y nivel de participación en el Proyecto MAPCOM (cont.)

\begin{tabular}{lccccc}
\hline & $\begin{array}{c}\text { He participado } \\
\text { en los } \\
\text { equipos por } \\
\text { áreas }\end{array}$ & $\begin{array}{c}\text { He sido } \\
\text { invitado a } \\
\text { participar o } \\
\text { me han } \\
\text { consultado en } \\
\text { la recopilación } \\
\text { de datos }\end{array}$ & $\begin{array}{c}\text { No he } \\
\text { participado }\end{array}$ & $\begin{array}{c}\text { Sin } \\
\text { contestación }\end{array}$ & Total \\
\hline Sin & 1 & 1 & & & \\
contestación & $11,1 \%$ & $11,1 \%$ & 0 & 7 & 9 \\
& $1,7 \%$ & $0,6 \%$ & $0,0 \%$ & $77,8 \%$ & $100,0 \%$ \\
& $0,1 \%$ & $0,1 \%$ & $0,0 \%$ & $0,8 \%$ & $1,1 \%$ \\
\hline Usuario web & 20 & 24 & 37 & 0 & 81 \\
& $24,7 \%$ & $29,6 \%$ & $45,7 \%$ & $0,0 \%$ & $100,0 \%$ \\
& $34,5 \%$ & $13,4 \%$ & $6,2 \%$ & $0,0 \%$ & $9,7 \%$ \\
& $2,4 \%$ & $2,9 \%$ & $4,4 \%$ & $0,0 \%$ & $9,7 \%$ \\
\hline Total & 58 & 179 & 592 & 9 & 838 \\
& $6,9 \%$ & $21,4 \%$ & $70,6 \%$ & $1,1 \%$ & $100,0 \%$ \\
& $100,0 \%$ & $100,0 \%$ & $100,0 \%$ & $100,0 \%$ & $100,0 \%$ \\
& $6,9 \%$ & $21,4 \%$ & $70,6 \%$ & $1,1 \%$ & $100,0 \%$ \\
\hline
\end{tabular}

En concreto, puede advertirse que el $89,3 \%$ no es usuario de la web $\mathrm{MapCom}$ y el $70,6 \%$ no ha participado en ninguno de los trabajos de la investigación. A hora bien, de los que han manifestado haber participado de alguna manera en los trabajos de $\mathrm{M}$ apCom formando parte de equipos o de los invitados a proporcionar datos, la mayoría (el 63,8\%) no ha hecho uso de la web, y solo el $48 \%(34,5 \%+13,4 \%)$ dice haber hecho uso de ella.

En conclusión, el interés mostrado al contestar la encuesta no se debe al conocimiento previo sobre este proyecto.

2. Perfiles de los investigadores y adscripción profesional y académica.

De los 838 encuestados, un $14 \%$ no son todavía doctores y son investigadores en formación, un $40 \%$ tiene una antigüedad de 10 a 30 años como doctor y solo un 3\% tiene antigüedad de más de 30 años. 
Por CC. A A ., Valencia, M adrid y A ndalucía están por encima del $14 \%$ en la proporción de doctorandos que contestan la encuesta. Por universidades, la U I I destaca tanto por el porcentaje de doctorandos que contestan la encuesta en la Comunidad Val enciana (un $63 \%$ ) como en la proporción de investigadores de la misma universidad que la contestan (un $43 \%$ ). La U CM también destaca por la proporción de doctorandos que contestan la encuesta tanto en la Comunidad de M adrid $(69,6 \%)$ como en la propia UCM $(29,1 \%)$.

Si tomamos en cuenta los sexenios concedidos que son declarados por los encuestados (pues el 15,6\% no contesta esta pregunta) es de resaltar, dada la representatividad de la muestra, que más de la mitad de nuestros investigadores (un $51,1 \%$ ) carece de sexenios, el $15,5 \%$ tiene uno, el $11,3 \%$ solo tiene dos, y con tres sexenios o más, solo un $6 \%$. Podría pensarse que estos datos están relacionados únicamente con la edad de nuestro universo de investigadores, pero no es así. Del rango de 31 a 40 años, el $79 \%$ carece de sexenios; del intervalo de 41 a 50 años, el $48 \%$ también carece de sexenios y, por último, del intervalo de 51 a 60 años, el $46 \%$ también carece de sexenios.

A la pregunta sobre el número de veces que el encuestado ha tenido experiencia como IP, un $24,6 \%$ no la contesta. De los que la contestan, un $44,4 \%$ responde que «nunca», un 14,3\% que «al menos una vez», y un $12,5 \%$ «de 2 a 5 veces». La experiencia, entonces, de una actividad continuada como IP resulta excepcional, pues con más de 5 ocasiones en calidad de IP no llegan al 5\%. Haber participado, en cambio, como miembro en equipos de investigación es algo más frecuente, si bien todavía un 16,9\% deja esta pregunta sin responder y un $8,1 \%$ contesta que «nunca». A hora bien, más de la mitad, un $56,3 \%$, ha participado de esta experiencia al menos una vez ( $18 \%$ ) y de 2 a 5 veces un $38,3 \%$.

Otro rasgo en la experiencia investigadora es haber dirigido tesis doctoral es y TFM, pregunta que casi cuatro de cada diez encuestados dejan sin responder, y que, entre los que la contestan, un 33,9\% dice que «nunca» ha dirigido una tesis doctoral y un 14,6\% que «nunca» ha dirigido un TFM o un TFG, grados estos últimos cuya dirección con más frecuencia (por encima de 10 veces) ha sido objeto de experiencia investigadora para la mitad de los encuestados.

A I invitar a los encuestados a que manifiesten si las valoraciones recibidas por sus actividades investigadoras en general (en evaluaciones de proyectos, artículos, tesis, etc.) les parecen «muy relevantes», «bastante relevantes» 0 «poco relevantes» en aqueIlo que atañe tanto a la innovación teórica o metodológica como a sus aplicaciones sociales, 0 al reconocimiento obtenido por citas y reseñas, destaca la renuncia a expresar valoraciones por parte de más de la mitad de los IP, mientras que cuatro de cada diez entre quienes responden en calidad de miembros de equipos de investigación tienden a 
considerar «bastante rel evantes» Ias valoraciones recibidas por los conceptos reseñados. R eteniendo los datos que se muestran en la Tabla 4, se advierten perfiles que anulan la supuesta oposición cuando se comparan las respuestas que, en calidad de IP, de miembros de equipos de investigación o de directores académicos dan los encuestados ante las valoraciones solicitadas que oponen el interés de las aplicaciones sociales de la investigación al interés personal del reconocimiento académico. Y perfiles similares aparecen cuando se comparan las respuestas por CC. A A . y universidades.

A las preguntas sobre uso y consulta de registros internacionales y nacionales de investigación, destaca en todos los casos el «uso» (más del $60 \%$ de los encuestados) sobre el «no uso» (menos del 20\%), si bien la frecuencia más repetida de consulta es la de «alguna vez» (en torno a un $30 \%$ de los encuestados), y la pregunta queda sin contestación entre un $20 \%$ y un $25 \%$ de los casos. Cinco de cada diez encuestados deja sin

\section{TABLA 4}

Respuestas sobre val oraciones recibidas por las actividades investigadoras en general (en evaluaciones de proyectos, artículos, tesis, etc.)

\section{Por Enfoques teóricos y metodológicos novedosos}

$\begin{array}{lccccc} & & & & \\ \end{array}$


TABLA 4

R espuestas sobre valoraciones recibidas por las actividades investigadoras en general (en evaluaciones de proyectos, artículos, tesis, etc.) (cont.)

Por Interés de sus aplicaciones a la sociedad

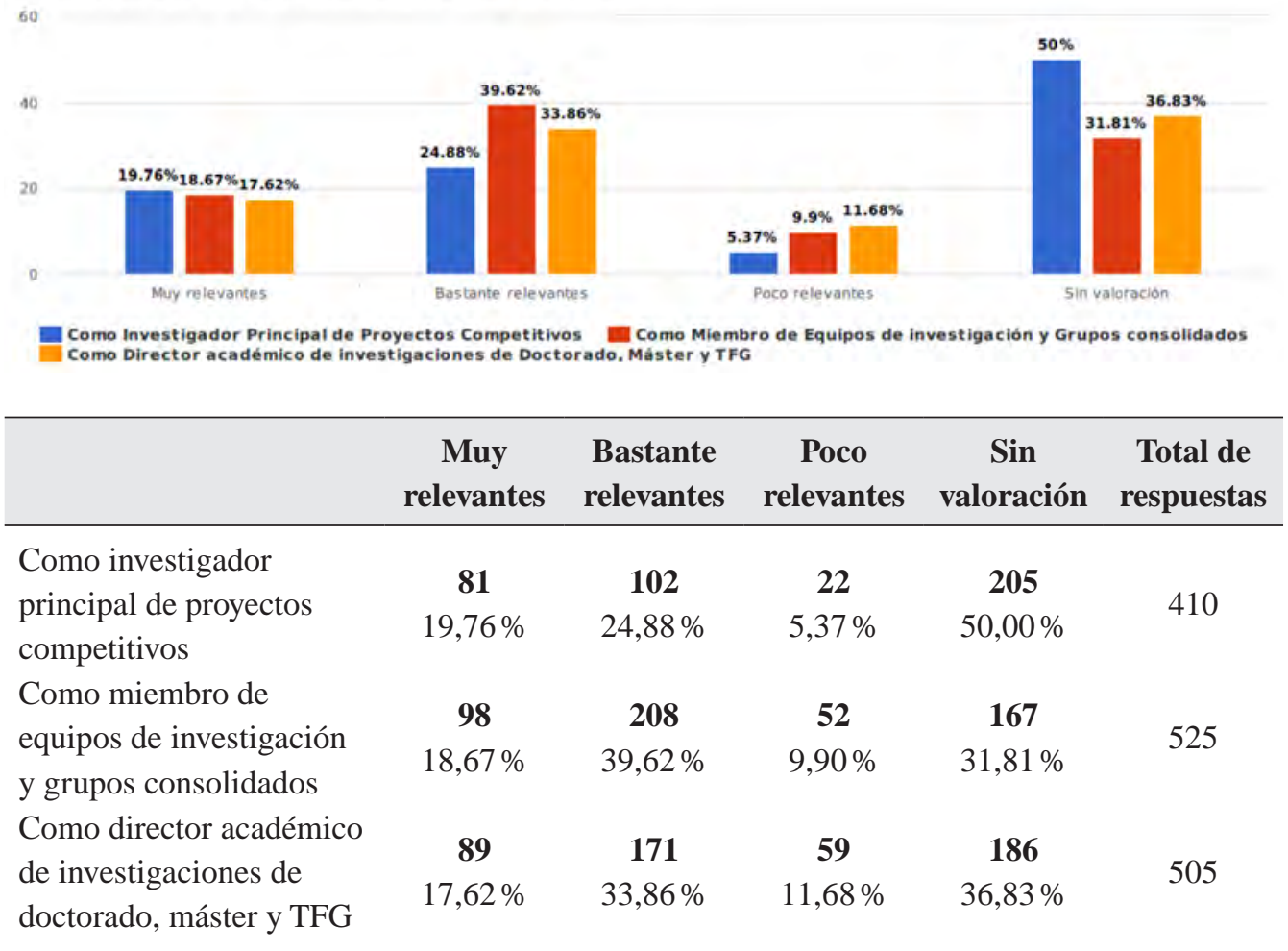

contestar la pregunta sobre participación en sociedades científicas, tanto nacionales como internacionales, y manifiesta no usarlas casi el $30 \%$, sobre todo si se trata de sociedades internacionales.

A la pregunta sobre cuál es la adscripción en función de la cual el encuestado se reconoce integrado en el censo de investigadores, cuatro de cada diez contesta que exclusivamente por su vinculación con una universidad, dos de cada diez por su vinculación a una universidad y a una sociedad científica, y en igual proporción dejan la pregunta sin contestar. La condición de estar adscrito a un programa de doctorado o a una sociedad científica sin ser doctor suma un $14 \%$. A la pregunta sobre el rango académico actual del encuestado, 4 de cada 10 manifiestan vinculación contractual indefinida (20,8\%) o temporal $(21,2 \%)$, mientras que los titulares y catedráticos, sean interinos o de plantilla, suman otros cuatro de cada diez, si bien los catedráticos apenas llegan al 6,3\%. 
Por CC. A A., y reparando solo en aquellas cuyo número de encuestados está por encima de la media, se observan los siguientes perfiles:

- En A ndalucía, cuatro de cada diez encuestados manifiestan vinculación contractual, y, entre los contractuales, destacan los de contratación temporal (36\%) frente a un $11,7 \%$ de contratación indefinida. Tres de cada diez son funcionarios, si bien $27,3 \%$ son titulares y $11,7 \%$ catedráticos. La UMA destaca en A ndalucía por el carácter contractual de los encuestados (más de un $50 \%$ ) y por el carácter temporal de los contratos: un $44 \%$.

- En Cataluña, el perfil mayoritario de los encuestados (más de la mitad) manifiesta natural eza contractual en su vinculación académica, si bien domina la indefinida $(37,2 \%)$ sobre la temporal $(25,5 \%)$, destacando la U OC y la URV por sumar el $80 \%$ de encuestados vinculados contractualmente, aunque la mayoría con contratación indefinida en ambas universidades.

- En la Comunidad de M adrid la mitad de los encuestados manifiesta también vinculación contractual, si bien un 30,1\% de carácter indefinido y un 21,2\% temporal; por su parte, los funcionarios que la contestan son titulares el $27,4 \%$ y catedráticos el 6,2\%. Entre sus universidades, la URJC destaca por invertir la proporción de los encuestados que manifiestan vinculación funcionarial (más de la mitad), pues son titulares el $48,1 \%$ y catedráticos el $7,7 \%$, y vinculación contractual el $42,4 \%$, la mitad de los cuales son indefinidos $(21,2 \%$ ) y la otra mitad temporales $(21,2 \%)$.

- En la Comunidad Valenciana casi el $60 \%$ de los encuestados manifiesta vinculación contractual, siendo $19 \%$ indefinida y $38 \%$ temporal, proporción que se comparte en sus universidades, con las excepciones de la UA y U CH/CEU, donde la proporción se invierte con 36,4\% indefinidos y 27,3\% temporales, respectivamente, en la primera, y con el $100 \%$ de indefinidos en la segunda.

- Finalmente, en Castilla y L eón cinco de cada diez encuestados manifiestan vinculación contractual (18\% indefinida y 32,8\% temporal) y dos de cada diez son funcionarios (14,8\% titulares y $9,8 \%$ catedráticos), proporciones compartidas por la mayor parte de la universidades de la comunidad salvo por la USA L, que aparece con mayor número de encuestados que son funcionarios ( $25 \%$ titulares y $6,2 \%$ catedráticos), frente a $6,2 \%$ y $12,5 \%$, que son contratados indefinidos y temporales respectivamente, y brindando un $50 \%$ de encuestados que son doctorandos.

Con tales perfiles sobre rangos académicos de los encuestados, cuya muestra por estratos de CC. AA . y universidades debemos reiterar que es representativa del censo, 
no es de extrañar la escasa experiencia investigadora en proyectos I+D, pues las convocatorias estatales de proyectos imponen como requisito que en los equipos de investigación deban figurar profesores con vinculación permanente (funcionarial o contractual) mientras duran los proyectos, excluyendo así a investigadores con vinculación temporal. En consecuencia, también resulta explicable la carencia o reducido número de sexenios reconocidos por la actividad investigadora.

\section{Docencia de los I nvestigadores}

U no de los conflictos que apareció reiteradamente en el discurso de las discusiones que se celebraron en $M$ adrid, B arcel ona y M álaga mediante la técnica del Phillips 66 fue el que aqueja al reconocimiento académico de la dedicación docente en detrimento de la actividad investigadora que, sin embargo, se requiere para que sea promovida la carrera universitaria. La actividad investigadora, de hecho, se halla bajo la presión de evaluaciones puntuales para competir en convocatorias de proyectos de muy diversa naturaleza y criterio, y de evaluaciones periódicas exigidas para acceder a plazas y rangos académicos, sin que la dedicación a la investigación sea reconocida en igualdad de condiciones que la docencia, y sin que los criterios de evaluación de la calidad investigadora consideren la excelencia de sus resultados por el avance de conocimiento y por retornos sociales, sino exclusivamente por el impacto de las publicaciones conforme al número de citas entre las élites de investigadores. Por esta razón, si el tiempo que se le dedica a la docencia es en detrimento de la actividad investigadora, conocer cuál es la docencia que manifiestan los investigadores encuestados resulta relevante.

Los datos de la Tabla 5 muestran el resultado de las respuestas de los encuestados tomando solo en consideración los tipos de docencia.

TABLA 5

Docencia grado y posgrado

\begin{tabular}{lcc}
\hline & Frecuencia & Porcentaje \\
\hline Docencia grado y posgrado & 375 & 44,7 \\
Sin contestación & 199 & 23,7 \\
Sin docencia & 73 & 8,7 \\
Solo docencia grado & 178 & 21,2 \\
Solo docencia posgrado & 13 & 1,6 \\
Total & 838 & 100,0 \\
\hline
\end{tabular}


En general, lo más relevante es que sumando «docencia grado y posgrado», «solo docencia grado» y «solo docencia posgrado», un $67,5 \%$ de los encuestados tienen docencia. Casi la mitad de los encuestados (el $44,7 \%$ ) comparte su docencia en grado y posgrado, uno de cada cinco solo tiene docencia en al gún grado y tan solo un 1,6\% la tiene exclusivamente en al gún posgrado.

A hora bien, agrupando las respuestas a las preguntas sobre docencia que se imparte tanto en grados como en posgrados, y diferenciando si esa docencia se centra prioritariamente en Periodismo (PER), en Comunicación A udiovisual (CAU), en Publicidad y RR. PP. (PUB) y en Documentación (DOC), Ios datos que se presentan en la Tabla 6 son relevantes, porque un $45 \%$ de los encuestados dice impartir docencia en el grado o posgrado de Periodismo (PER). U na tercera parte de los encuestados deja la pregunta sin contestar cuando la docencia por la que se pregunta es en grado o posgrado de Periodismo, y cinco de cada diez encuestados manifiesta que en Periodismo no tiene docencia.

Si se pregunta por la docencia en Comunicación A udiovisual (CAU), un 39,6\% dice impartirla en grado o posgrado, otro $37,9 \%$ no contesta esta pregunta y, finalmente, cinco de cada diez encuestados manifiestan que en CAU no tienen docencia.

Si se pregunta por la docencia en Publicidad y RR. PP. (PUB), un 31,2\% dice impartirla en grado o posgrado, un $41,5 \%$ no contesta a esta pregunta y un $27,2 \%$ declara no tener docencia en PUB.

Por último, si se pregunta por la docencia en Documentación (DOC) un 5,4\% dice impartirla en grado o posgrado, un 52,6\% no contesta esta pregunta, y un 42,0\% manifiesta que no tiene docencia en DOC.

TABLA 6

Docencia grado y posgrado cuatro titulaciones

\begin{tabular}{lrrrrrrrr}
\hline & \multicolumn{2}{c}{ PER } & \multicolumn{2}{c}{ CAU } & \multicolumn{2}{c}{ PUB } & \multicolumn{2}{c}{ DOC } \\
\hline & Frec. & $\%$ & Frec. & $\%$ & Frec. & $\%$ & Frec. & $\%$ \\
\hline Docencia grado y posgrado & 211 & 25,2 & 136 & 16,2 & 126 & 15,0 & 20 & 2,4 \\
Sin contestación & 281 & 33,5 & 318 & 37,9 & 348 & 41,5 & 441 & 52,6 \\
Sin docencia & 159 & 19,0 & 188 & 22,4 & 228 & 27,2 & 352 & 42,0 \\
Solo docencia grado & 161 & 19,2 & 162 & 19,3 & 116 & 13,8 & 11 & 1,3 \\
Solo docencia posgrado & 26 & 3,1 & 34 & 4,1 & 20 & 2,4 & 14 & 1,7 \\
Total & 838 & 100 & 838 & 100 & 838 & 100 & 838 & 100 \\
\hline
\end{tabular}

Tales datos revelan que la docencia exclusiva en posgrado, que sería la más cercana a las exigencias de la actividad investigadora, es absolutamente minoritaria para todas 
las titulaciones, de forma que lo más frecuente es que si hay docencia en posgrado también sea compartida con el grado, presumiblemente perjudicando así la dedicación a la actividad investigadora. M ás si se tiene en cuenta que la docencia exclusiva en grado es mayor porcentual mente en PER y CAU (cinco de cada diez encuestados), algo menor en PUB, y es en DOC donde la docencia exclusiva en grado presenta menos casos. Pero, además, es en DOC donde hay ligeramente más casos en docencia de posgrado que en grado y, sobre todo, un $42 \%$ de los que contestan la pregunta declara no tener docencia. A unque también es en esta titulación donde más encuestados no contestan a la pregunta.

\section{Experiencia personal en la actividad investigadora}

\section{a) La integración en equipos de investigación}

A la pregunta sobre integración del encuestado en grupos y equipos de investigación, no responde el $29,4 \%$ de los encuestados y, entre los que responden, el 15,8\% carece de adscripción a proyectos vigentes, mientras que manifiesta su integración en ellos el 40,3\%, declarando estar incorporado al grupo de investigación el 31,6\%, al equipo de trabajo que caracteriza a investigadores con vinculación contractual el 8,7\%, y en calidad de IP solo el $14,6 \%$.

Como es sabido, los equipos y grupos de proyectos son efímeros, pues su duración suele ser la misma que la del proyecto, de tres años en su mayoría. Supuestamente, los equi pos que cada universidad reconoce como grupos de investigación consolidados (GIC) conservan una vigencia de larga duración y en su historial se pueden suceder variedad de proyectos a lo largo del tiempo, tanto de convocatorias estatales como autonómicas, de universidad y de contratos con terceros mediando el artículo 83, por ejemplo, para regular desde las universidades los trámites de contratación con empresas e instituciones. Pues bien, cuando se les ha preguntado a los encuestados por su integración en GIC, el 30,3\% no responde y el $12,5 \%$ declara no estar integrado en ningún grupo consolidado de investigación. A sí pues, una mayoría que roza el $55 \%$ declara su participación en los GIC, en calidad de IP un $9,2 \%$ y en calidad de miembro de los equipos de trabajo o del grupo de investigadores un $6,4 \%$ y un $41,5 \%$, respectivamente. De todos modos, 430 encuestados (un $51,3 \%$ ) facilitaron al menos un nombre o una U RL del propio grupo de investigación.

A las preguntas sobre participación en diferentes categorías de proyectos, unos internacionales, otros nacionales de convocatorias estatales, autonómicas y competitivas de universidad, y, final mente por contratos regulados por el artículo 83, citado con anterioridad, los datos más rel evantes cuyo comentario excusamos son estos: 
- Si se trata de proyectos internacionales, el $46,5 \%$ no contesta la pregunta y el $33,3 \%$ dice que ninguno. Con alguna experiencia en ellos, un 12,1\% declara uno solo, de modo que con más de uno apenas se llega al $8 \%$ de los encuestados.

- Si se trata de proyectos nacionales de convocatorias estatales, el $41,4 \%$ no contesta la pregunta, y el $16,3 \%$ dice que ninguno. Con alguna experiencia suman el $42,3 \%$ en total.

- Si se trata de proyectos autonómicos, un $43,4 \%$ no contesta y un $22,7 \%$ dice que ninguno. Con alguna experiencia desde uno a más de seis, aparece un total de $33,9 \%$.

- Si se trata de proyectos de universidad, un $45 \%$ no contesta la pregunta y dice carecer de ellos un 21,8\%. Con alguna experiencia desde uno a más de seis, aparece un total de $33,3 \%$, muy similar al del apartado anterior.

\section{TABLA 7}

Referencias de fila y columna según el orden de las palabras clave de las líneas de investigación por campos de estudio

\begin{tabular}{|c|c|c|c|c|c|c|}
\hline $\begin{array}{l}\text { CAMPOS DE } \\
\text { ESTUDIO }\end{array}$ & $\begin{array}{l}\text { Comunicación } \\
\text { mediática }\end{array}$ & $\begin{array}{l}\text { Comunicación } \\
\text { organizacional }\end{array}$ & $\begin{array}{l}\text { Comunicación } \\
\text { de grupos }\end{array}$ & $\begin{array}{l}\text { Comunicación } \\
\text { interpersonal }\end{array}$ & $\begin{array}{c}\text { Teoría y } \\
\text { episte-mología }\end{array}$ & $\begin{array}{l}\text { Métodos y } \\
\text { técnicas }\end{array}$ \\
\hline $\begin{array}{l}\text { Comunicación } \\
\text { mediática }\end{array}$ & $\begin{array}{l}\text { Com. mediática } \\
\text { general }\end{array}$ & $\begin{array}{l}\text { Com. mediática } \\
\text { organización }\end{array}$ & $\begin{array}{l}\text { Com. mediática } \\
\text { de grupos }\end{array}$ & $\begin{array}{l}\text { Com. mediática } \\
\text { interpersonal }\end{array}$ & $\begin{array}{l}\text { Com. } \\
\text { mediática de } \\
\text { teorías }\end{array}$ & $\begin{array}{l}\text { Com. } \\
\text { mediática de } \\
\text { métodos }\end{array}$ \\
\hline $\begin{array}{l}\text { Comunicación } \\
\text { organizacional }\end{array}$ & $\begin{array}{l}\text { Com. } \\
\text { organizacional } \\
\text { mediática }\end{array}$ & $\begin{array}{l}\text { Com. } \\
\text { organizacional } \\
\text { general }\end{array}$ & $\begin{array}{l}\text { Com. } \\
\text { organizacional } \\
\text { de grupos }\end{array}$ & $\begin{array}{l}\text { Com. } \\
\text { organizacional } \\
\text { interpersonal }\end{array}$ & $\begin{array}{l}\text { Com. } \\
\text { organizacional } \\
\text { de teorías }\end{array}$ & $\begin{array}{l}\text { Com. } \\
\text { organizacional } \\
\text { de métodos }\end{array}$ \\
\hline $\begin{array}{l}\text { Comunicación } \\
\text { de grupos }\end{array}$ & $\begin{array}{l}\text { Com. grupal } \\
\text { mediática }\end{array}$ & $\begin{array}{l}\text { Com. grupal } \\
\text { organización }\end{array}$ & $\begin{array}{l}\text { Com. grupal } \\
\text { general }\end{array}$ & $\begin{array}{l}\text { Com. grupal } \\
\text { interpersonal }\end{array}$ & $\begin{array}{l}\text { Com. grupal } \\
\text { de teorías }\end{array}$ & $\begin{array}{l}\text { Com. grupal } \\
\text { de métodos }\end{array}$ \\
\hline $\begin{array}{l}\text { Comunicación } \\
\text { interpersonal }\end{array}$ & $\begin{array}{l}\text { Com. } \\
\text { interpersonal } \\
\text { mediática }\end{array}$ & $\begin{array}{l}\text { Com. } \\
\text { interpersonal } \\
\text { organizaciones }\end{array}$ & $\begin{array}{l}\text { Com. } \\
\text { interpersonal } \\
\text { de grupos }\end{array}$ & $\begin{array}{l}\text { Com. } \\
\text { interpersonal } \\
\text { general }\end{array}$ & $\begin{array}{l}\text { Com. } \\
\text { interpersonal } \\
\text { de teorías }\end{array}$ & $\begin{array}{l}\text { Com. } \\
\text { interpersonal } \\
\text { de métodos }\end{array}$ \\
\hline $\begin{array}{l}\text { Teoría y } \\
\text { epistemología }\end{array}$ & $\begin{array}{l}\text { Teorías com. } \\
\text { mediáticas }\end{array}$ & $\begin{array}{l}\text { Teorías com. } \\
\text { organizacional }\end{array}$ & $\begin{array}{l}\text { Teorías com. } \\
\text { grupal }\end{array}$ & $\begin{array}{l}\text { Teorías com. } \\
\text { interpersonal }\end{array}$ & $\begin{array}{l}\text { Teorías com. } \\
\text { general }\end{array}$ & $\begin{array}{l}\text { Teoría de } \\
\text { métodos }\end{array}$ \\
\hline $\begin{array}{l}\text { Métodos y } \\
\text { técnicas }\end{array}$ & $\begin{array}{l}\text { Metodología } \\
\text { com. mediática }\end{array}$ & $\begin{array}{l}\text { M etodología } \\
\text { com. } \\
\text { organizacional }\end{array}$ & $\begin{array}{l}\text { M etodología } \\
\text { com. grupal }\end{array}$ & $\begin{array}{l}\text { M etodología de } \\
\text { com. } \\
\text { interpersonal }\end{array}$ & $\begin{array}{l}\text { M etodología } \\
\text { de las teorías }\end{array}$ & $\begin{array}{l}\text { M etodología } \\
\text { general }\end{array}$ \\
\hline
\end{tabular}

N ota: las categorías en rojo son aquellas con al menos un caso, y las categorías en morado las que carecen de casos 
- Finalmente, si se trata de proyectos por contratación mediante el artículo 83, no contesta la pregunta un $47,7 \%$ y carece de ellos $31,0 \%$. Con alguna experiencia desde uno a más de seis, aparece un total de $21,2 \%$.

L a encuesta proseguía abordando enseguida preguntas en torno a líneas de investigación, primero, y después, en torno a objetivos de la experiencia investigadora y objetos de estudio por campos temáticos.

A propósito de las líneas de investigación hay que destacar que 479 de los 838 encuestados (el 57,2\%) brindaron palabras clave para describir líneas de investigación. Como se trataba de una pregunta con respuestas abiertas, la columna cuyos datos recogían las palabras clave de las líneas de investigación hubo de ser recodificada. Las siguientes 26 categorías que figuran en la Tabla 7 sirvieron para esta recodificación. L os tipos en color en las casillas de la tabla expresan el cruce de las referencias de fila y columna siguiendo el orden con que se sucedían las palabras clave de las líneas de investigación por campos.

b) La fijación de objetivos, la elección de objetos de estudio y el tipo de técnicas empleadas para elaborar e interpretar datos

Conforme a la tabla anterior, los objetivos de la experiencia investigadora y objetos de estudio por campos temáticos quedan así dispuestos para su procesamiento y explotación cuya distribución de respuestas se recogen en la Tabla 8.

Debe ser resaltado que, a esta pregunta sobre objetivos en función de objetos de estudio por campos, más de la mitad de los encuestados prefieren no responder, salvo para la elección que consiste en «explicar» los rasgos de los objetos de estudio. No obstante, los encuestados comparten similar frecuencia en la el ección de objetivos de investigación sobre objetos de estudio mayoritariamente del campo de la comunicación mediática, siendo el más frecuente de estos objetivos el de «describir» y el menos frecuente el de «intervenir», de forma que, por los perfiles de estas respuestas, la experiencia investigadora se acomodaría más a los primeros desarrollos de una disciplina que al desarrollo característico de su madurez en aplicaciones sociales. En lo que atañe a las frecuencias resultantes de la recodificación de las líneas de investigación por campos, reiterando que solo 479 de los 838 encuestados (el 57,2\%) brindaron palabras clave para describir líneas de investigación, las frecuencias fueron las que aparecen en la Tabla 9. 


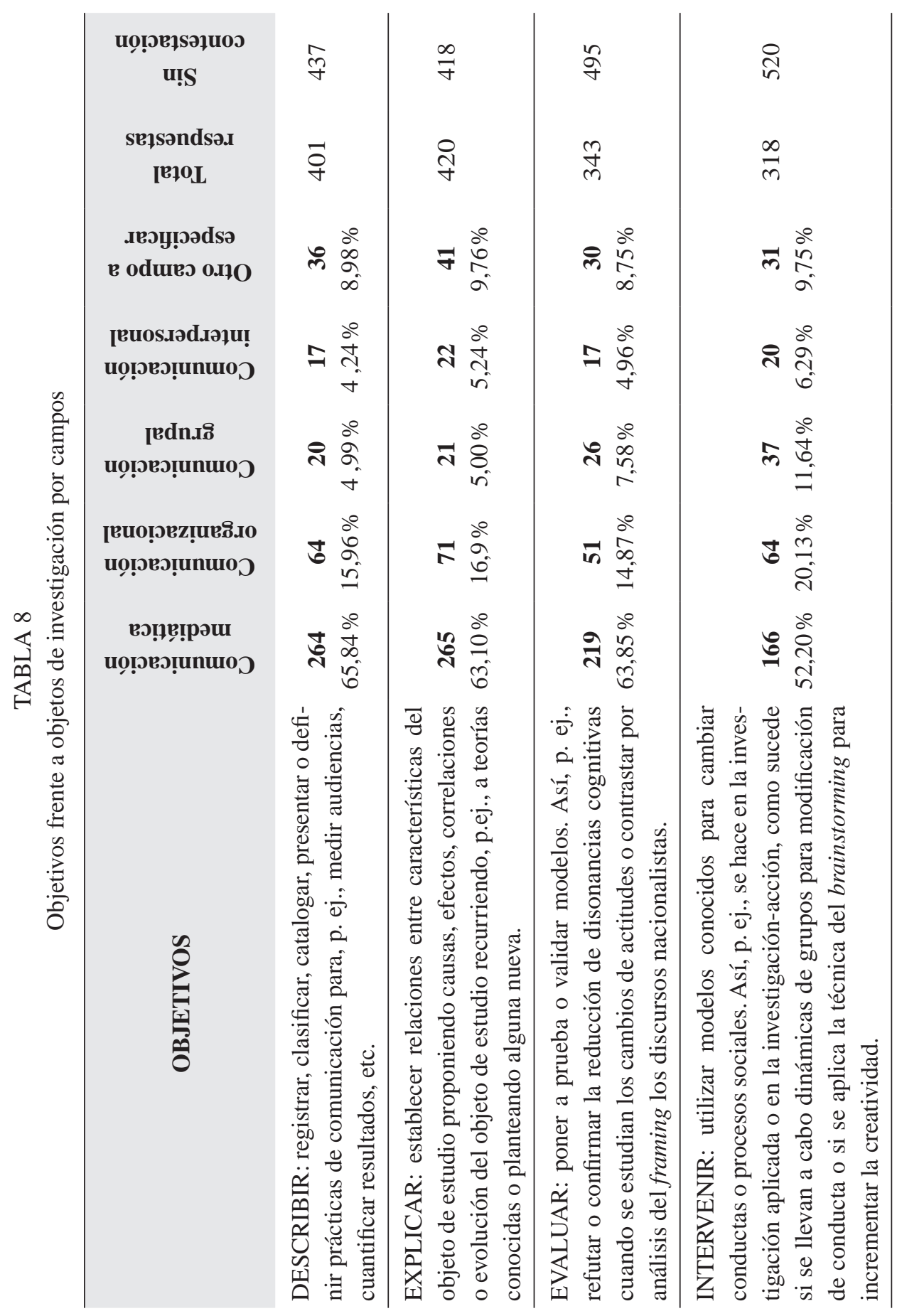


TABLA 9

Líneas de investigación por campos

\begin{tabular}{lcc}
\hline & Frecuencia & Porcentaje \\
\hline Com. grupal general & 1 &, 1 \\
Com. grupal mediática & 2 &, 2 \\
Com. interpersonal general & 1 &, 1 \\
Com. interpersonal mediática & 3 &, 4 \\
Com. mediática de grupos & 67 & 8,0 \\
Com. mediática general & 66 & 7,9 \\
Com. mediática interpersonal & 36 & 4,3 \\
Com. mediática organización & 132 & 15,8 \\
Com. organizacional general & 8 & 1,0 \\
Com. organizacional interpersonal & 3 &, 4 \\
Com. organizacional mediática & 96 & 11,5 \\
M etodología com. grupal & 2 &, 2 \\
M etodología com. mediática & 24 & 2,9 \\
M etodología com. organizacional & 1 &, 1 \\
M etodología general & 7 &, 8 \\
Sin contestación & 360 & 43,0 \\
Teorías com. General & 11 & 1,3 \\
Teorías com. Grupal & 1 &, 1 \\
Teorías com. interpersonal & 2 &, 2 \\
Teorías com. mediáticas & 14 & 1,7 \\
Teorías com. organizacional & 1 &, 1 \\
Total & 838 & $\mathbf{1 0 0 , 0}$ \\
\hline
\end{tabular}

A la vista, pues, de estos resultados, destacan las líneas de investigación cuyo interés gira sobre la comunicación mediática por naturaleza de su organización, con un $15 \%$ de apariciones, seguidas de aquellas líneas de investigación centradas sobre las resonancias mediáticas de la comunicación organizacional, con una frecuencia del $11,5 \%$, así como sobre la comunicación mediática de grupos, con $8,0 \%$ y, en fin, sobre la comunicación mediática en sus propias referencias mediáticas, con un 7,9\%. Tal atención prestada al campo de la comunicación mediática corrobora los datos que con similares proporciones resultaron del análisis de contenido de la producción de tesis y proyectos I+D en los años comprendidos entre 2007 y 2013, inclusive. A specto este que, al ser comentado en las sesiones del Phillips 66, mereció una explicación compartida en casi todos los grupos de debate: el origen de nuestras facultades universitarias 


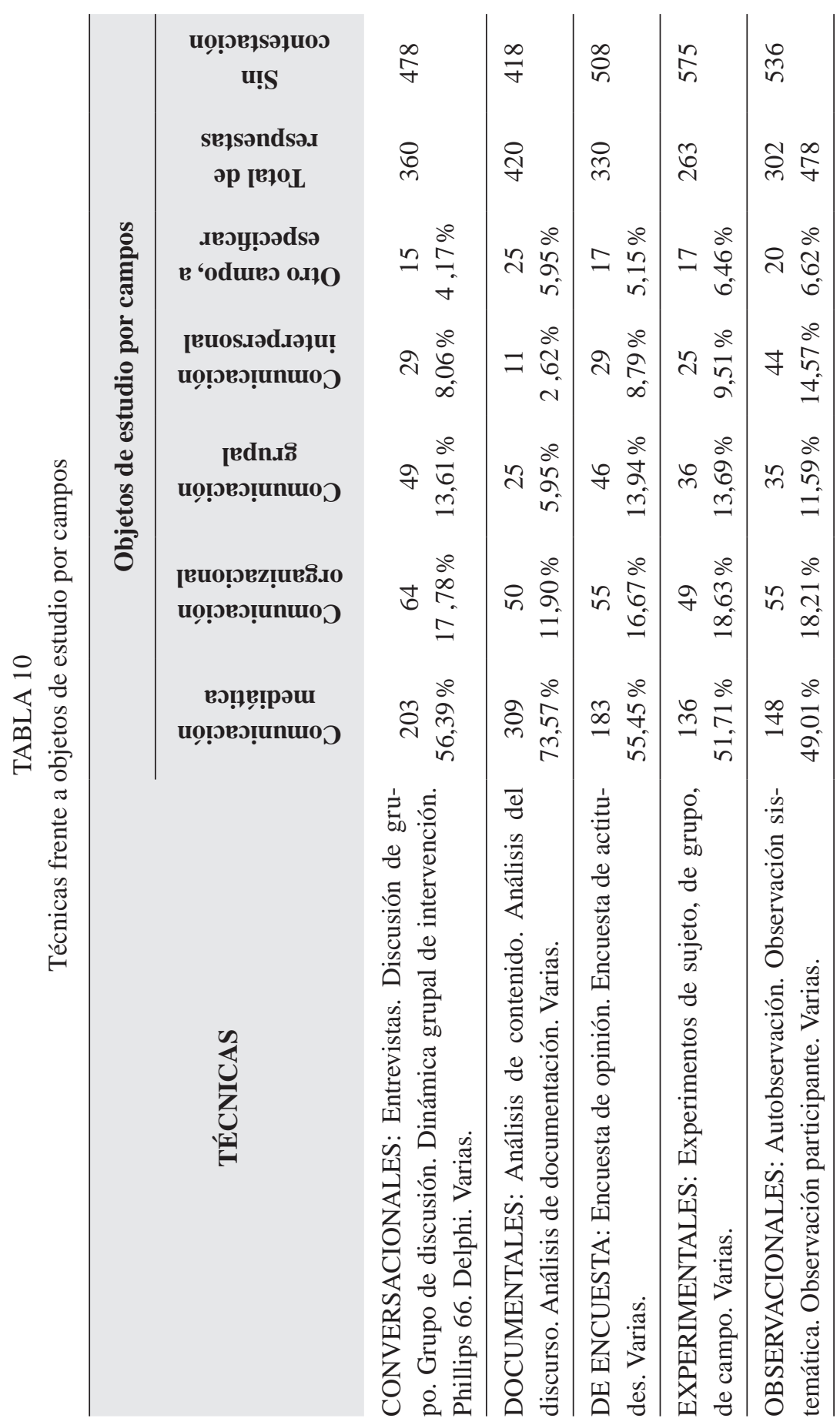


fue orientado a cumplir la función social de la formación superior de profesionales para los medios, y no para otras prácticas sociales de comunicación tales como, por ejemplo, la salud, la educación, la política o la empresa. Por otra parte, cuando se habla hoy día de comunicación mediática, se incluye en este rubro las prácticas mediadoras del universo digital.

Si ahora se fija la atención en cuáles son las técnicas que los encuestados señalan como las más utilizadas con relación a los objetos de estudio por los campos que han sido el egidos en su experiencia investigadora, se vuelve a repetir la prioridad del objeto de estudio integrado en el campo de la comunicación mediática, siendo para este menester la técnica de análisis documental es (análisis de contenido, análisis del discurso, análisis de documentación) la más utilizada. Pero vuelve a sorprender que más de la mitad de los encuestados dejen la pregunta sin responder cuando se trata de indicar técnicas de investigación el egidas para abordar los objetos de estudio por campos, cual esquiera que sean estos, si bien algo más de la mitad señala la elección de las técnicas de análisis documentales.

L os datos sobre las preguntas a propósito de las técnicas elegidas en la investigación sobre los objetos de estudio por campos vienen también a confirmar que la experiencia investigadora se expresa con limitaciones graves posiblemente debidas a que su dedicación carece de créditos académicos, la gran parte de ellos a favor de la dedicación docente, lo que impide a su disciplina el desarrollo propio de una madurez a la que todos aspiramos.

\section{c) La valoración de la experiencia investigadora en relación con el beneficio obtenido como retorno}

A la hora de valorar la propia experiencia investigadora referida al beneficio obtenido como retorno, primero se preguntó por las revistas indexadas y no indexadas en ciencias sociales. La Tabla 11 permite comparar los datos sobre las valoraciones al ternativas atribuidas por los encuestados a las revistas indexadas y no indexadas; más de 390 no respondieron a estas preguntas, que, en todo caso, fueron contestadas por un número que osciló entre 370 y 390 encuestados.

Después se preguntó por las ediciones de libros y monografías y sobre las actas de congresos. L a Tabla 12 permite comparar los datos sobre las valoraciones aplicadas por los encuestados a las ediciones de libros y monografías y a las actas de congresos. También en este caso las preguntas fueron contestadas por un número que osciló de 370 a 390 encuestados. 
Tabla 11

Valoración sobre cuánto influyen en la investigación los criterios de publicación en las revistas indexadas y no indexadas

Revistas indexadas en CC. Sociales (p.e. JCR, Scopus, Scholar Metrics, etc.) influyen:

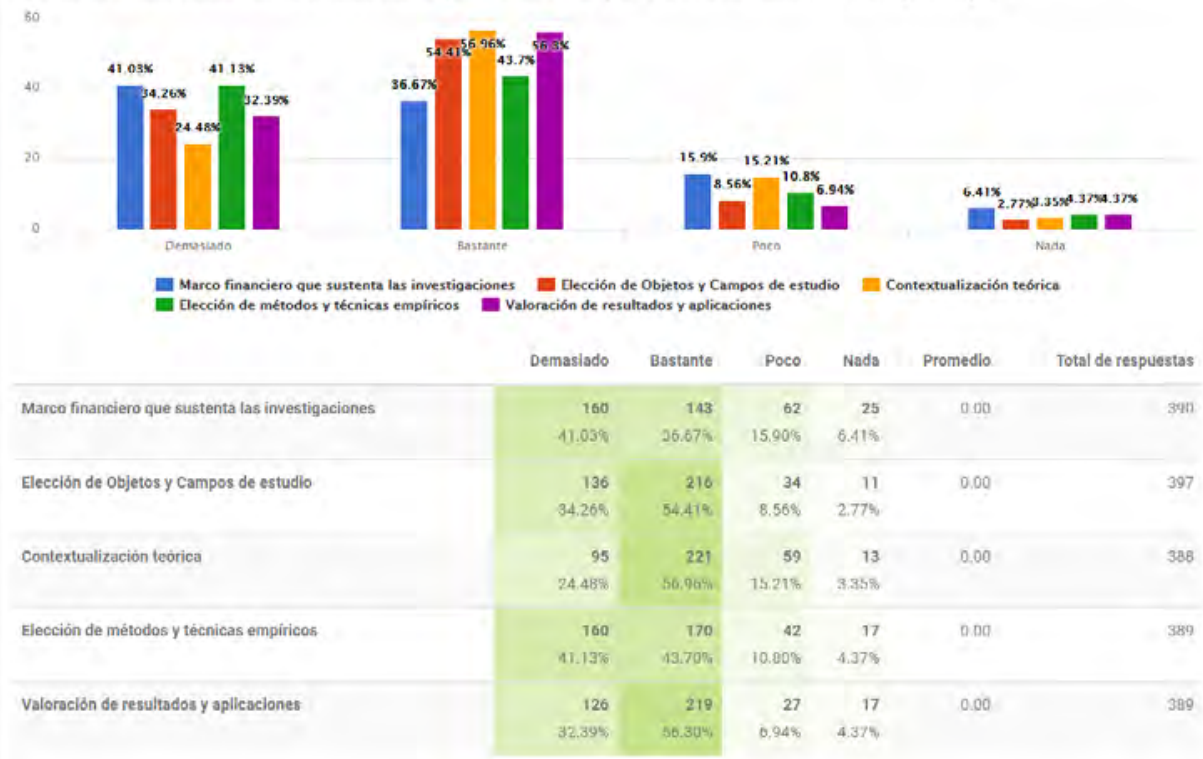

Revistas no indexadas en Comunicación influyen:

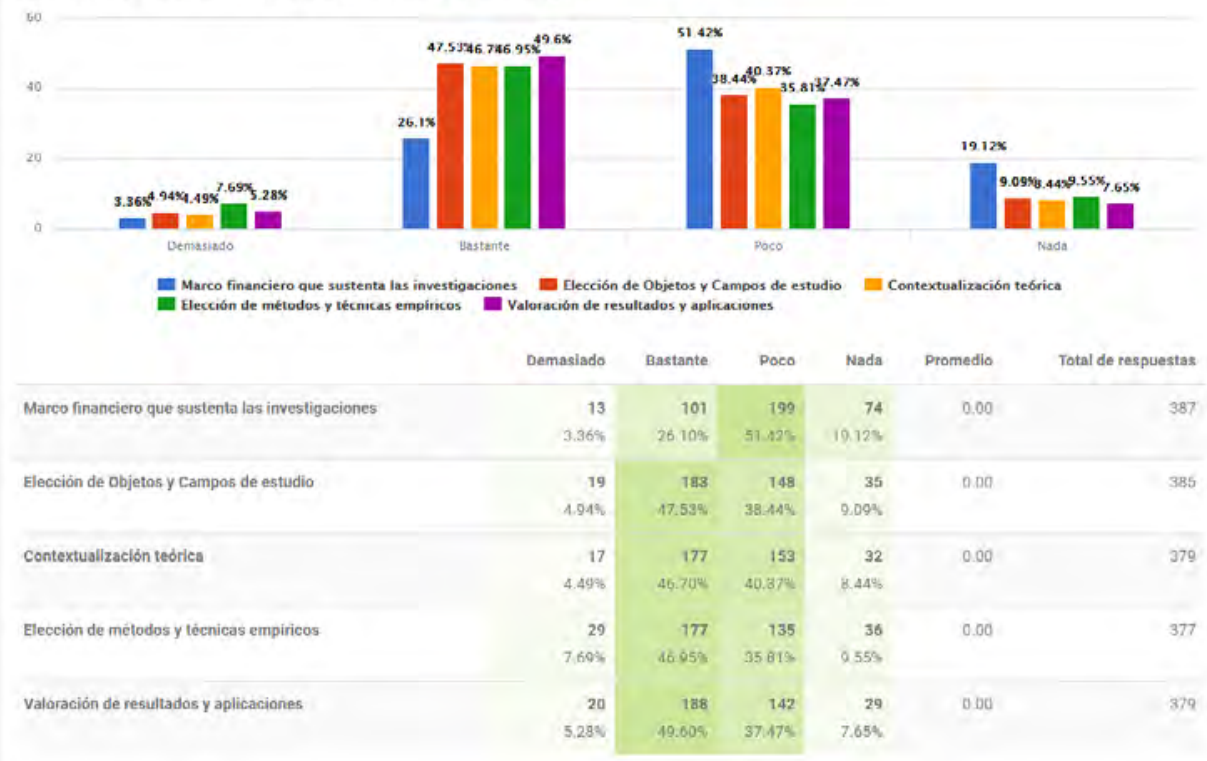




\section{TABLA 12}

Valoración sobre cuánto influyen en la investigación los criterios de publicación en las ediciones de libros y monografías y en actas de congresos

Ediciones de libros y monografias influyen:

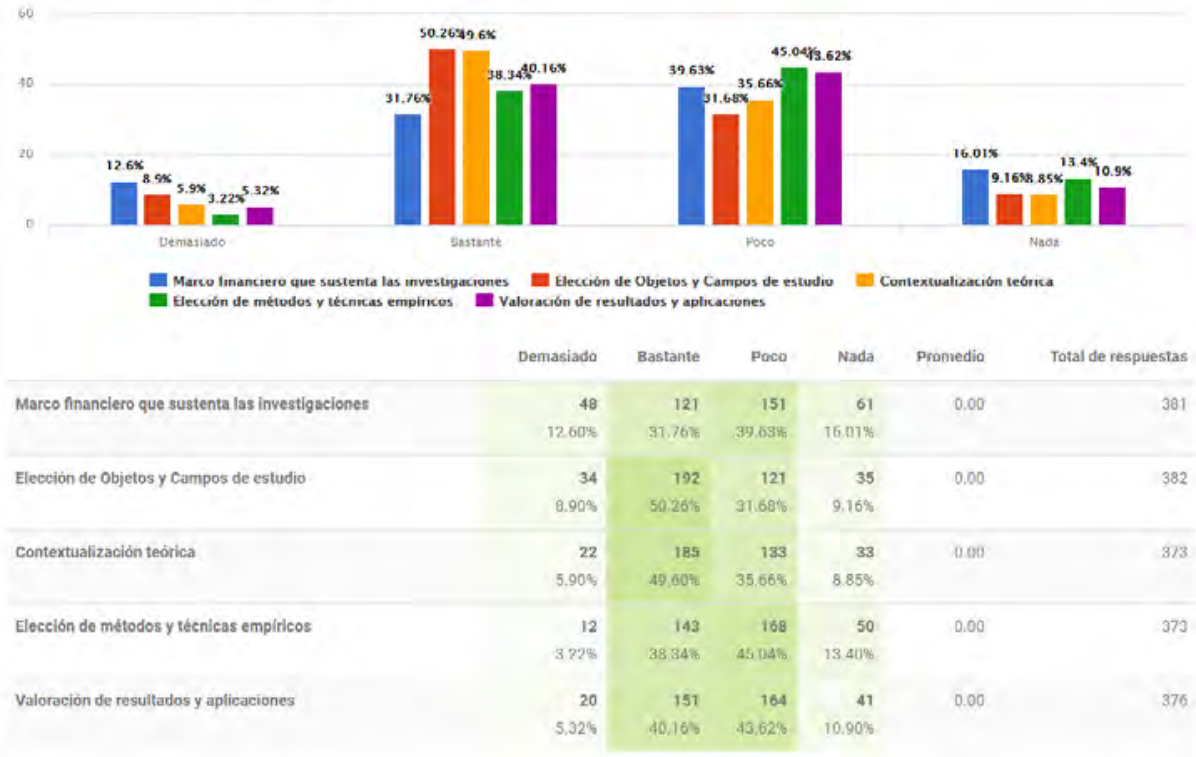

Actas de Congresos influyen:

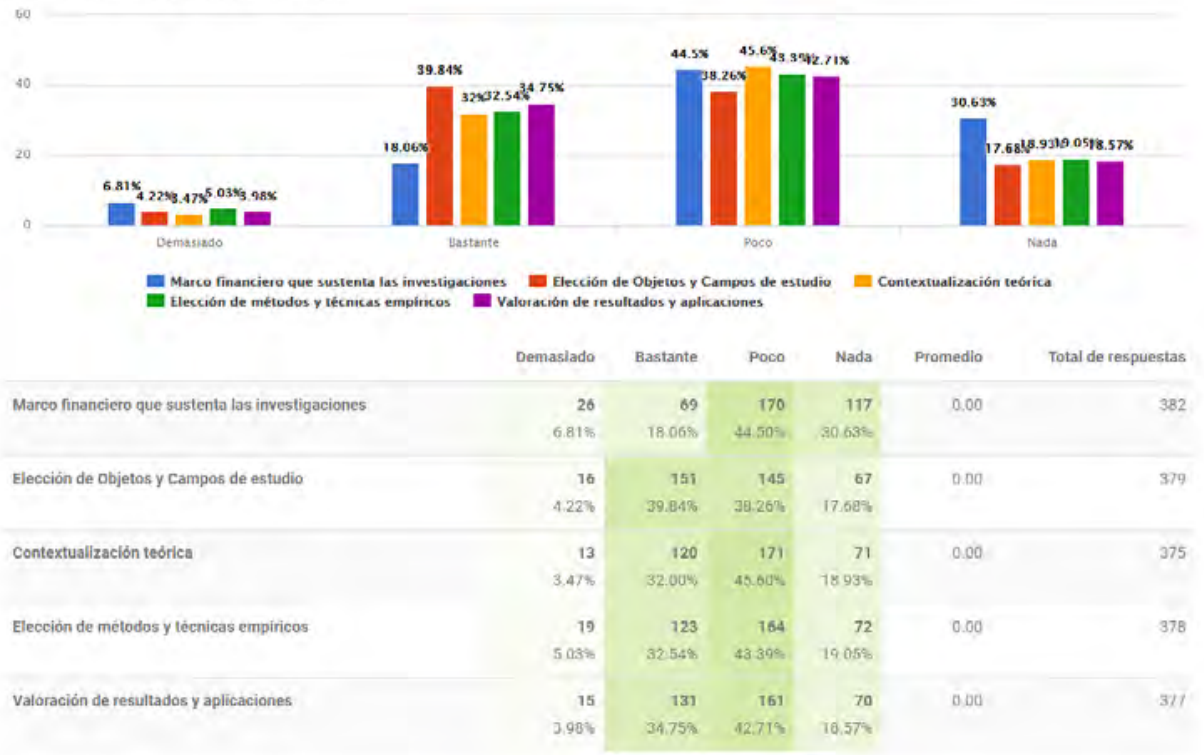


Los datos son reveladores. A las revistas se les atribuyen mayor influencia que a los libros y monografías y que a las actas de los congresos, sobre todo por los criterios de marcos financieros y de elección de objetos y campos de estudio en primer lugar, y por los criterios de contextualización teórica en segundo lugar, puesto que este criterio sobresale cuando es valorado «no demasiado».

Las ediciones de libros y monografías, así como las actas de congresos, resultan menos valorados, pero especialmente reteniendo los criterios de elección de objetos y campos de estudio y de elección de métodos y técnicas.

A sí pues, por una parte, se constata una visión crítica sobre rendimientos de la investigación en lo que atañe a la publicación de resultados, de los cuales se valoran menos sus aplicaciones que el marco financiero que los sustenta; por otra parte, si se trata de las revistas de mayor impacto, se constata especialmente la elección de objetos y campos de estudio.

También, al objeto de valorar la propia experiencia investigadora referida al beneficio obtenido como retorno, se preguntó en segundo lugar por la dimensión del retorno 0 el rendimiento dominante en función de los objetivos que ha perseguido la investigación. La Tabla 13 permite comparar los datos sobre los diferentes rendimientos dominantes obtenidos en relación con los objetivos perseguidos por la actividad investigadora. $L$ as publicaciones y ediciones vuelven a ser citadas como rendimiento mayoritariamente dominante en general, pero, sobre todo, para los objetivos de «explicar» y «evaluar», así como algo menos citadas la presentación en congresos. El retorno por divulgación/formación ocupa un tercer rango.

Se puede resal tar también que el total de respuestas a esta pregunta es notablemente inferior al número de respuestas a las preguntas inmediatamente anteriores, pues el número de encuestados que la responden oscila entre un mínimo de 284 y un máximo de 364.

O tro de los rendimientos de la experiencia investigadora por los que se les preguntó a los encuestados tenía que ser referido a los rendimientos que sus publicaciones e impactos de investigación produjeron en sus trayectorias profesionales, detallando " «contratos para empresas o instituciones», «movilidad: estancias y becas», «evaluaciones académicas: sexenios», y «acreditación académica: doctorado, titularidad, cátedra, etc.». En la Tabla 14 se ofrecen los gráficos con las valoraciones expresadas por los encuestados sobre la importancia atribuida a tales rendimientos.

A la vista de la comparación de los datos en la valoración de tales rendimientos en la trayectoria profesional de los encuestados, aparece que mayoritariamente se valoran como «nada importante» las contrataciones externas al ámbito universitario así como las evaluaciones académicas referidas a los sexenios, mientras que las acreditaciones 


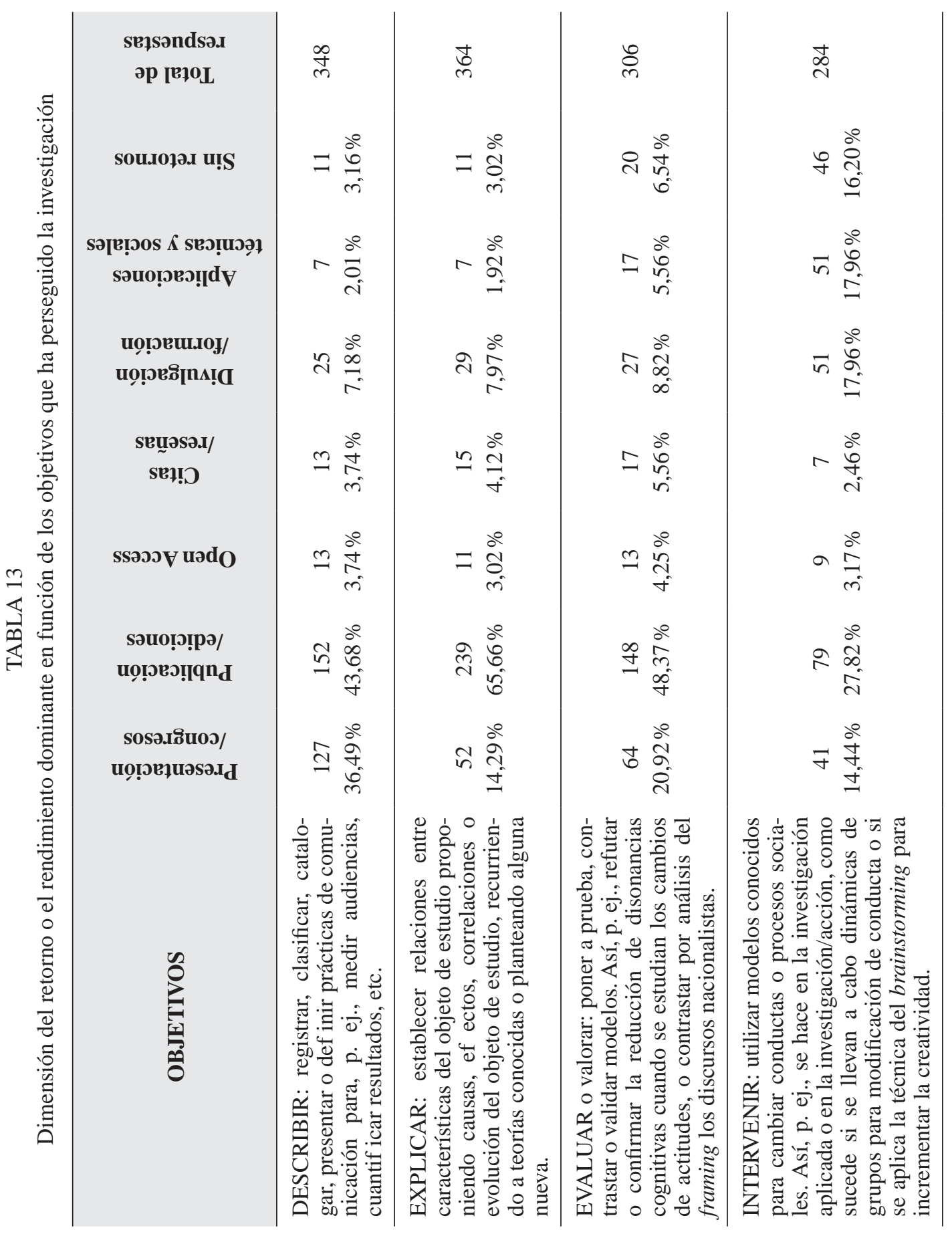


académicas, por el contrario, aparecen mayoritariamente valoradas como rendimiento «muy importante», y que la movilidad académica subvencionada es vista también por una mayoría simple como rendimiento «bastante importante» en la trayectoria profesional. Este es otro aspecto que viene a corroborar también que, en las trayectorias profesionales, la vida universitaria y la vida socioeconómica externa se dan la espal da, y que los rendimientos más valorados de la experiencia investigadora están orientados a la movilidad esencialmente académica.

\section{TABLA 14}

Rendimientos de impactos de investigación sobre trayectorias profesionales

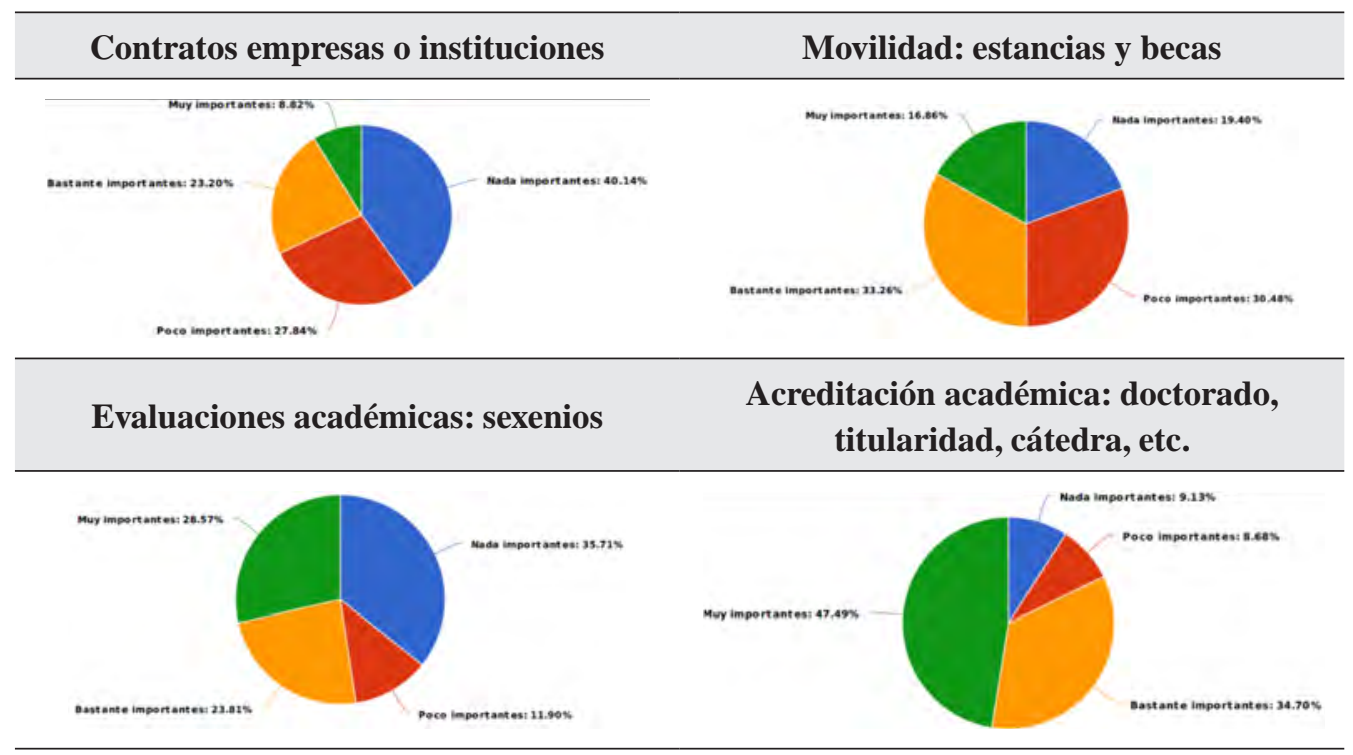

d) Valoración de las condiciones materiales, organizacionales e institucionales que según la experiencia de los encuestados afectan en España a la actividad investigadora

Prosiguió la encuesta preguntando a los encuestados su valoración a propósito de las debilidades y fortalezas de la investigación en comunicación, al valorar negativa o positivamente las condiciones materiales, organizacionales e institucional es a las que, según su experiencia, ha estado sometida en España la actividad investigadora. Para ello, el formulario invitaba a puntuar cada ítem conforme a una escal a de L ikert, desde -3 como 
extremadamente negativo hasta +3 como extremadamente positivo. L as Tablas 15, 16 y 17 permiten contemplar la comparación de valoraciones por ítem.

A I apreciar las condiciones de los marcos institucionales referidos a la compatibilidad de investigación y docencia, a las relaciones entre universidad y empresa, 0 a las agencias de evaluación y a la calidad de congresos y participación, aparecen mayoritariamente valoraciones positivas solo al juzgar la calidad de congresos y participación, mientras que son más frecuentes las valoraciones extremadamente negativas (-3) al juzgar la compatibilidad entre investigación y docencia, las relaciones entre universidad y empresa y al juzgar las agencias de evaluación ANECA, A NEP y CNEAI.

A la vista de estos perfiles se puede colegir que, para más de la mitad de los investigadores, pues contestan esta pregunta más del $50 \%$ de los encuestados, las condiciones materiales que acompañan a los procesos de investigación son valoradas negativamente, salvo al referirse a instalaciones y laboratorios, cuya valoración es en conjunto positiva.

TABLA 15

Debilidades y fortalezas de la investigación en comunicación al valorar las CONDICIONES MATERIALES

\begin{tabular}{|c|c|c|c|c|c|}
\hline \multicolumn{3}{|c|}{$\begin{array}{l}\text { Subvenciones y financiación últimos } \\
\qquad 10 \text { años }\end{array}$} & \multicolumn{3}{|c|}{ Becas y contratos últimos 10 años } \\
\hline Respuesto & Porcematose & comitios & - Respuesto & Porcentsito & Comitidad \\
\hline 3 & ${ }^{27 \cdots} \square$ & 119 & 3 & ${ }^{2346 x} \square$ & 100 \\
\hline 2 & ${ }_{18455}^{\square}$ & s & .2 & ${ }_{20065} \square$ & 92 \\
\hline. & $15 \sin$ & $\infty$ &. & 14ats & os \\
\hline - & +2 & 6 &. & ${ }^{14955} \mathbf{D}$ & as \\
\hline . & 19ass & $\omega$ & $\cdot \cdot$ & ${ }^{16635} \square$ & $n$ \\
\hline .2 & 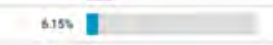 & ${ }_{27}$ & .2 & 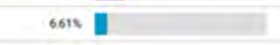 & 29 \\
\hline \multirow[t]{2}{*}{3} & $\sin 1$ & 14 & $\cdot 3$ & $\sin 1$ & 14 \\
\hline & Totaldecteroventar & 439 & & Totalderespoustas & 439 \\
\hline \multicolumn{3}{|c|}{ Trámites burocráticos últimos 10 años } & \multicolumn{3}{|c|}{$\begin{array}{l}\text { Instalaciones y laboratorios últimos } \\
\qquad 10 \text { años }\end{array}$} \\
\hline Respuerte & Porrentale & commitiod & Respuesta & Percemalo & cantidad \\
\hline 3 & ${ }^{34.785}=$ & 152 & 3 & 17415 & 74 \\
\hline 2 & ${ }_{1390} \mathrm{E}$ & 76 & 2 & ${ }^{11295}$ 回 & 48 \\
\hline 1 & 1304s & 5 & 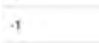 & 10.763 & $\$$ \\
\hline - & 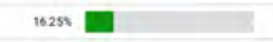 & $\pi$ & - & 26825 & 114 \\
\hline$\cdot \rightarrow$ & ${ }^{10985}$ & 48 & $*$ & ${ }^{1882 x} \square$ & $\infty$ \\
\hline .2 & 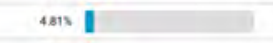 & ${ }^{21}$ & .2 & $8 \pi 1$ & 3 \\
\hline \multirow[t]{2}{*}{.3} & 27851 & 12 & $\cdot 3$ & sise & 22 \\
\hline & Total de cespuestsos & 437 & & Total de respoestas & 425 \\
\hline
\end{tabular}


TABLA 16

Debilidades y fortalezas de la investigación en comunicación, al valorar las CONDICIONES ORGANIZACIONALES

\begin{tabular}{|c|c|c|c|c|c|}
\hline \multicolumn{3}{|c|}{$\begin{array}{l}\text { Requisitos exigidos en convocatorias } \\
\text { públicas }\end{array}$} & \multicolumn{3}{|c|}{ Programas de formación metodológica } \\
\hline Reepouts & Parcentale & comitioses & Respuents & Porcentale & comilisad \\
\hline 3 & ${ }^{18525}$ 口 & so & 3 & 20705 & 89 \\
\hline .2 & 19215 & ${ }^{83}$ & -2 & ${ }_{16985}^{\square}$ & 73 \\
\hline-1 & 19685 & as & $\rightarrow$ & 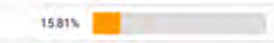 & 68 \\
\hline 0 & ${ }^{18755} \square$ & * & $\circ$ & 25.125 & 108 \\
\hline+1 & 15515 & 67 & $*$ & ${ }^{14,42 \times}$ & 62 \\
\hline$*^{2}$ & 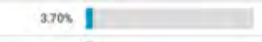 & 16 & +2 & 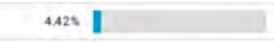 & 19 \\
\hline \multirow[t]{2}{*}{$\cdot 3$} & $4 \sin 1$ & ${ }^{20}$ & .3 & $250 \times 1$ & $"$ \\
\hline & Total de repposets: & 432 & & Total derenowatis & 430 \\
\hline \multicolumn{3}{|c|}{ Redes de cooperación y conocimiento } & \multicolumn{3}{|c|}{ Recursos humanos en los equipos } \\
\hline Respesests & Porcentalo & canitioad & Respousto & Porcentale & contisased \\
\hline-3 & 9.185 & ${ }^{30}$ & 3 & ${ }_{12185}$ & 32 \\
\hline .2 & 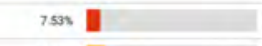 & 32 & -2 & ${ }^{10.300} \mathbf{E}$ & 4 \\
\hline-1 & 10.5m & 45 & + & ${ }^{13585}$ & so \\
\hline 0 & ${ }^{26.355} \square$ & 112 & i & $26.00 \mathrm{E}$ & $m$ \\
\hline+1 & ${ }^{29.185}$ & 124 & + & ${ }^{2506 x} \square$ & 107 \\
\hline+2 & ${ }^{14.125} \square$ & $\infty$ & +2 & 9.135 & 39 \\
\hline \multirow[t]{2}{*}{+3} & + & 13 & +3 & 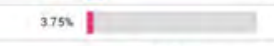 & 16 \\
\hline & Totalde respuestas & 425 & & Total de respuestas & 427 \\
\hline
\end{tabular}

En cambio, la tendencia a la valoración negativa no es la dominante para los aspectos propuestos a ser juzgados en lo que respecta a condiciones organizacionales de los procesos de investigación. Así, si las valoraciones positivas aparecen menos al juzgar los requisitos exigidos en convocatorias públicas y los programas de formación metodológica, las valoraciones positivas aparecen en mayor medida cuando se aprecian las redes de cooperación y conocimiento y los recursos humanos en los equipos.

Finalmente, aunque no es una condición institucional más, el rol que desempeñan las revistas científicas y los efectos de su indexación, al objeto de poder comparar el perfil de su valoración entre los encuestados con el perfil de la valoración de los otros marcos institucionales, se incluyó este ítem junto a los demás referidos a las condiciones institucionales, y este perfil aparece en la Tabla 18, donde vuelve a incluirse el perfil de la valoración de congresos y participación junto al de la valoración de las revistas científicas y su indexación.

L os perfiles de la valoración que atañen a la calidad de congresos y su participación y a la indexación de las revistas científicas no suponen juzgar condiciones de los proce- 
TABLA 17

Debilidades y fortalezas de la investigación en comunicación, al valorar las CONDICIONES INSTITUCIONALES

\begin{tabular}{|c|c|c|c|c|c|}
\hline \multicolumn{3}{|c|}{ C ompatibilidad investigación y docencia } & \multicolumn{3}{|c|}{ R elaciones entre universidad y empresa } \\
\hline Respouts & Porcentaste & comitios & Respoutso & Porcentalo & conetiens \\
\hline 3 & 29685 & 130 & a & $24805 \square$ & 107 \\
\hline .2 & ${ }_{1735}$ & 70 & 2 & $17212 \mathbf{D}$ & 74 \\
\hline - & ${ }^{12350} \square$ & st & .1 & $1270 x+1$ & ss \\
\hline 0 & ${ }^{16675} \mathrm{E}$ & 73 & . & $23.72 \times$ & 102 \\
\hline$\bullet$ & ${ }^{13018} \mathbf{\square}$ & 5 & + & ${ }_{14.55}$ & \& \\
\hline .2 & 7oes ע & 3 & $2^{2}$ & sass I & 23 \\
\hline \multirow[t]{2}{*}{$\cdot 3$} & 38851 & ${ }_{17}$ & .3. & 1.0001 & - \\
\hline & Total deeresouestas & 43 & \multicolumn{3}{|c|}{ Totalderespuestas } \\
\hline \multicolumn{3}{|c|}{$\begin{array}{l}\text { Agencias de evaluación: ANE CA, ANE P, } \\
\text { CNEAI }\end{array}$} & \multicolumn{3}{|c|}{ Calidad de congresos y participación } \\
\hline Respousto & Portentalso & comitad & Resposesto & Porcentolote & comitised \\
\hline 3 & 27.925 & 122 & 3 & ${ }_{7825}$ & 34 \\
\hline .2 & 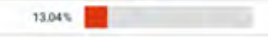 & 5 & $=2$ & 8sis = & 37 \\
\hline+ & 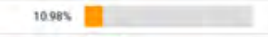 & 48 & $\rightarrow$ & $10575=$ & 46 \\
\hline - & ${ }^{21515} \square$ & is & 0 & ${ }_{21.615} \square$ & 94 \\
\hline 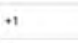 & ${ }^{15395} \square$ & 67 & +1 & $28515 \square$ & 124 \\
\hline+2 & 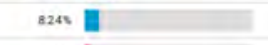 & 36 & +2 & 18.165 $\square$ & 79 \\
\hline \multirow[t]{2}{*}{+3} & 29751 & ${ }^{13}$ & .3 & 4835 & 21 \\
\hline & Total de respuestat & 43 & & & 435 \\
\hline
\end{tabular}

TABLA 18

Debilidades y fortalezas de la investigación en comunicación al valorar los M A RCOS INSTITUCIONA LES de congresos y la indexación de revistas científicas

\begin{tabular}{|c|c|c|c|c|c|}
\hline \multicolumn{3}{|c|}{ Calidad de congresos y participación } & \multicolumn{3}{|c|}{ Indexación de revistas científicas } \\
\hline Respuesta & Porcentalie & cambisad & Respoests & Porrentopte & Camitiad \\
\hline 3 & 7825 ] & ${ }^{34}$ & 3 & 14872 回 & os \\
\hline .2 & ess: - & $n$ & 2 & $\operatorname{sos} \|$ & 29 \\
\hline , & $1058 \times$ & 46 & 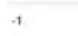 & 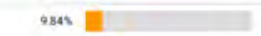 & 43 \\
\hline . & ${ }^{21618}$ & * & - & ${ }^{18765} \mathrm{D}$ & 12 \\
\hline+ & $28.510 \square$ & 124 & $\cdot$ & $2357 \times \square$ & 103 \\
\hline .2 & t8168 $\bar{\square}$ & 79 & $\cdot 2$ & ${ }_{18315}$ & $\infty$ \\
\hline \multirow[t]{2}{*}{+3} & 4825 & 21 & $\cdot 3$ & $\operatorname{sens} \overline{-1}$ & 3s \\
\hline & Total de respustas & 435 & & Total det respuestas & 437 \\
\hline
\end{tabular}

sos de investigación, sino valorar las valoraciones de sus resultados, es decir, metavalorar los marcos de su reconocimiento institucional. En este caso, esta metavaloración aparece muy mayoritariamente positiva. A sí pues, dado que, como expusimos al princi- 
pio, la muestra obtenida por el retorno de respuestas a la encuesta permite representarse significativamente al universo de los investigadores censados en centros de la universidad española con docencia reglada en Comunicación, se podría concluir que estos son menos críticos ante la metavaloración de su actividad investigadora que frente a las condiciones material es y organizacionales en las que la desarrollan. ¿Y cómo este censo de investigadores encaja dentro de la población universitaria cuando se retienen rasgos de edad, género, formación de origen e ingresos económicos? Terminaremos este informe con la exposición de tales datos.

\section{Perfiles sociodemográficos del censo de investigadores en comunicación respondiendo a las preguntas sobre edad, género, formación de origen y rango de ingresos económicos por la actividad profesional}

Los perfiles sociodemográficos del censo de investigadores en comunicación responden a intervalos de edad y género que, como se ve en la Tabla 19, reúnen a más del

TABLA 19

Perfiles sociodemográficos de edad y género

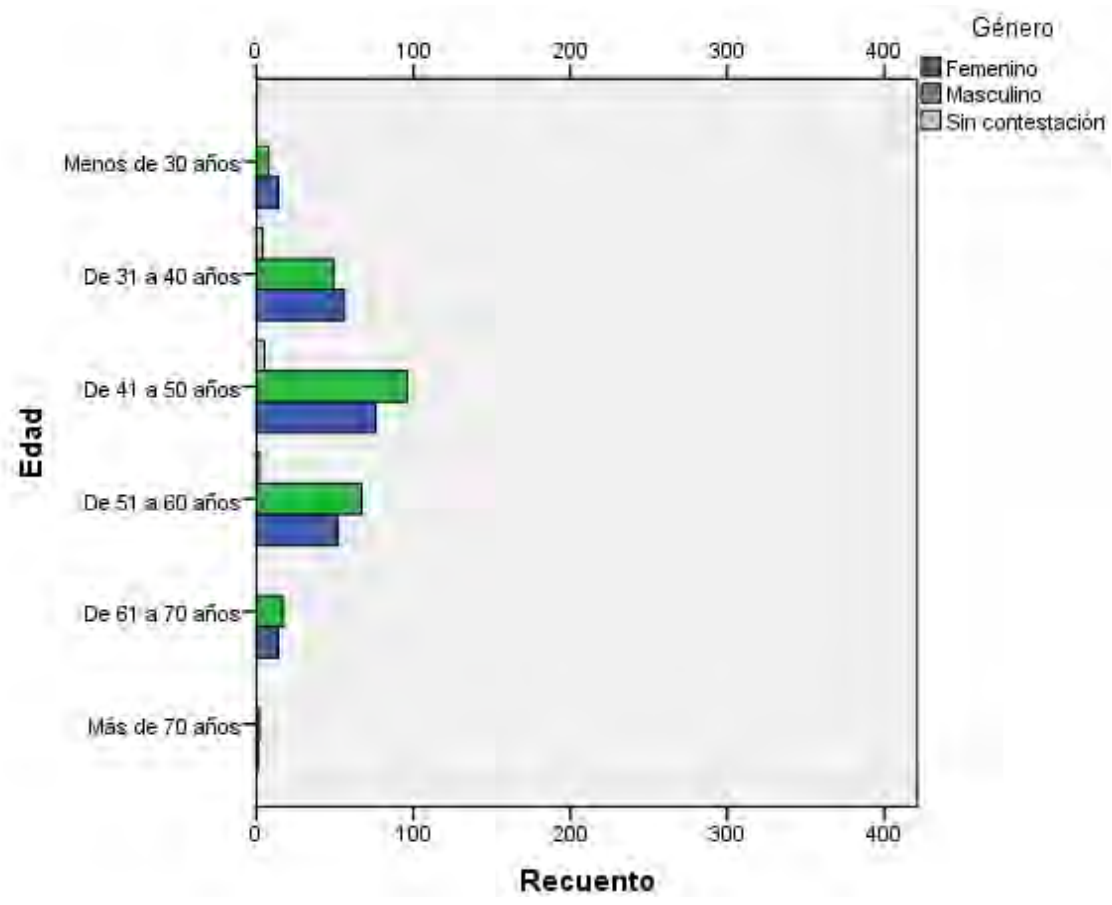


$50 \%$ de investigadores mayores de 41 años y menores de 60 . U na cuarta parte son menores de 40 años, y, entre los séniores o mayores de 61, apenas uno de cada 10. Por lo que, respecto a género, a medida que aumenta el rango de la edad disminuye la proporción de mujeres, y, cuanto menor es la edad, mayor es el número de estas. Hay un techo de cristal a partir de los 40 años. Como se puede comprobar si sobre la base de datos se cruzan estas variables por comunidades autónomas, se encuentran relevantes diferencias, entre las que sobresale la comunidad autónoma del País Vasco, cuya proporción de mujeres es siempre superior a la de hombres para todos los intervalos de edad.

En la Tabla 20 se ofrecen datos sobre la apreciación personal de la propia formación previa. Según se trate de humanidades, de ciencias sociales, de ciencias de la natural eza 0, finalmente, de matemáticas y tecnologías, los perfiles de la apreciación manifestada son diferentes.

En primer lugar, aparecen las humanidades y las ciencias sociales muy destacadas en su apreciación, tanto si la referencia es a la formación preuniversitaria como a la formación de grado y posgrado. En segundo lugar, aparecen las ciencias de la naturaleza y las matemáticas y tecnologías como las consideradas «no procedentes» si la referencia es a la formación de grado y posgrado.

Por otra parte, resulta relevante que casi una cuarta parte $(23,6 \%)$ considera las humanidades «no procedentes» si se trata de la formación de posgrado, mientras que para este mismo nivel de estudios las ciencias sociales casi nadie las considere «no procedentes», y se aprecie su formación como «excelente» para más de la mitad de los encuestados (un $51,76 \%$ ), sobre todo si se trata del posgrado.

De forma inversa, las ciencias de la naturaleza y las matemáticas y tecnologías son formaciones previas apreciadas como satisfactorias cuando se refieren al nivel preuniversitario, mientras que casi dos terceras partes de los encuestados las consideran «no procedentes» referidas a la formación de grado y de posgrado.

Con tales perfiles generales en la apreciación de la formación adquirida, no es de extrañar la dificultad que se impone a una política científica para transformar los diseños curriculares en beneficio de las ciencias experimentales, así como el esfuerzo añadido al que se enfrentan los académicos del ámbito de la comunicación para tener éxito en las evaluaciones a que someten sus trabajos para ser publicados en revistas cuyo impacto viene medido por parámetros habituales en el ámbito de las ciencias experimentales. Estas circunstancias vienen también a entorpecer las aspiraciones de los académicos de la comunicación para hacerse reconocer sus especialidades en los listados actuales de los códigos UNESCO y códigos NABS.

Finalmente, en las Tablas 21 y 22 aparecen datos sobre los ingresos profesionales ordenados por interval os de menor a mayor cuantía cada $1000 €$, y luego por CC. A A. 
TABLA 20

A preciación personal de la propia formación previa

Humanidades

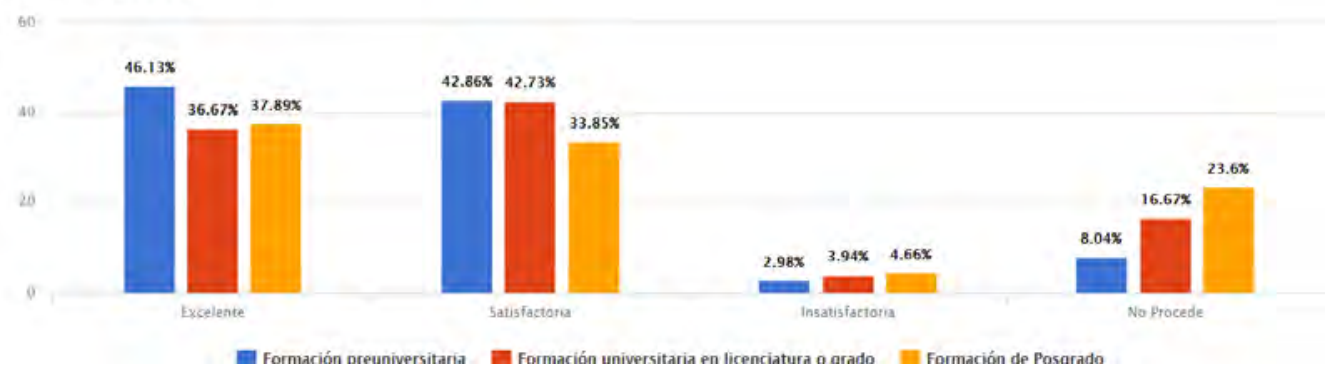

Ciencias sociales

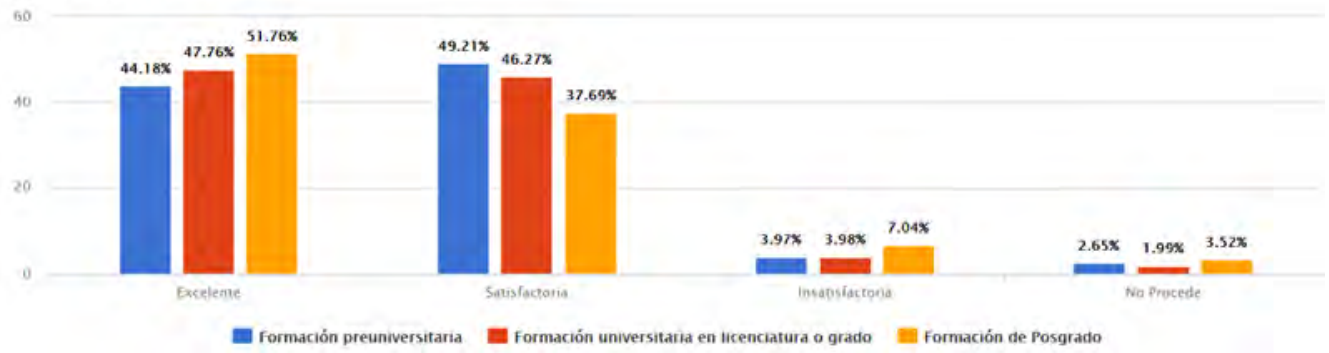

Ciencias de la naturaleza

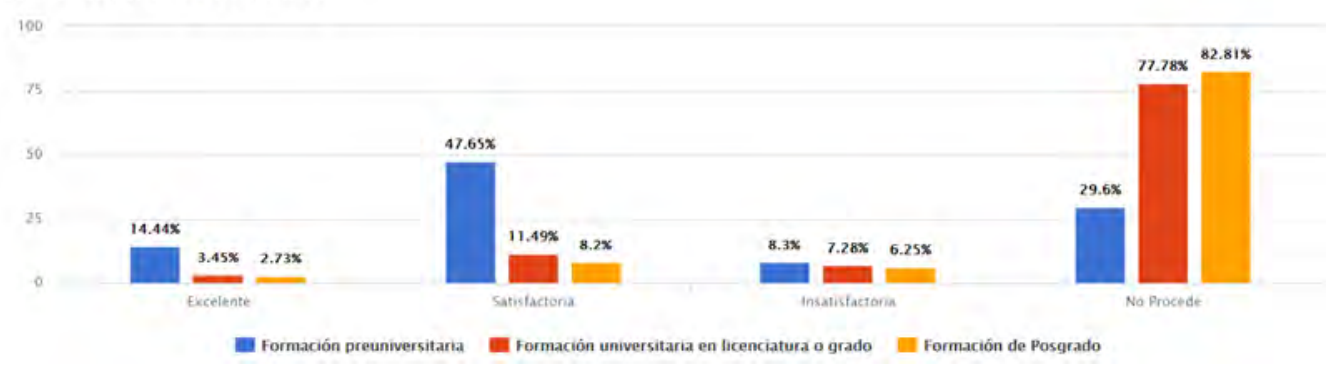

Matemáticas y tecnologías

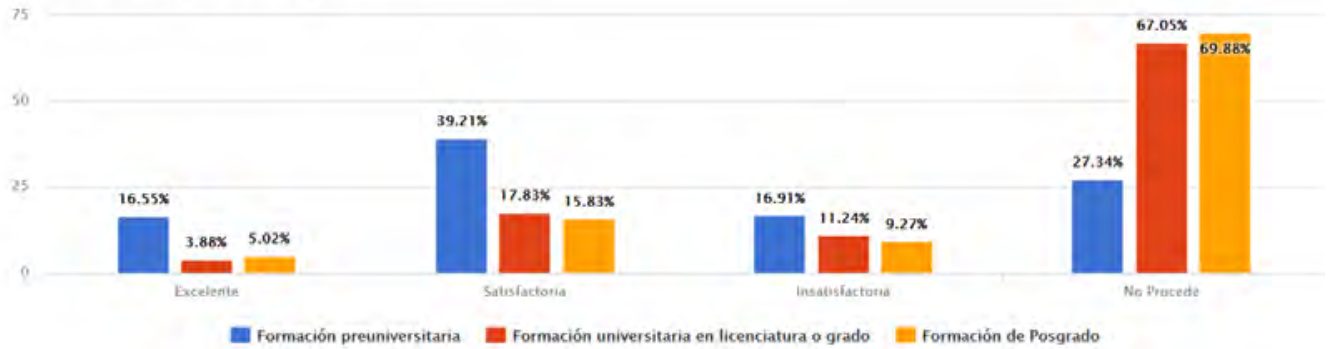


TABLA 21

Ingresos mensuales

\begin{tabular}{lcc}
\hline & Frecuencia & Porcentaje \\
\hline M enos de $1000 €$ & 68 & 8,1 \\
M ás de $1000 €$ y menos de $2000 €$ & 134 & 16,0 \\
Más de $10000 €$ & 13 & 1,6 \\
Más de $2000 €$ y menos de $3000 €$ & 151 & 18,0 \\
M ás de $3000 €$ y menos de $4000 €$ & 57 & 6,8 \\
Más de $4000 €$ y menos de $5000 €$ & 7 &, 8 \\
Más de $5000 €$ y menos de $6000 €$ & 3 &, 4 \\
Más de $6000 €$ & 4 &, 4 \\
Sin contestación & 401 & 47,9 \\
Total & 838 & 100,0 \\
\hline
\end{tabular}

TABLA 22

Gráficos de ingresos profesionales y por CC. A A.

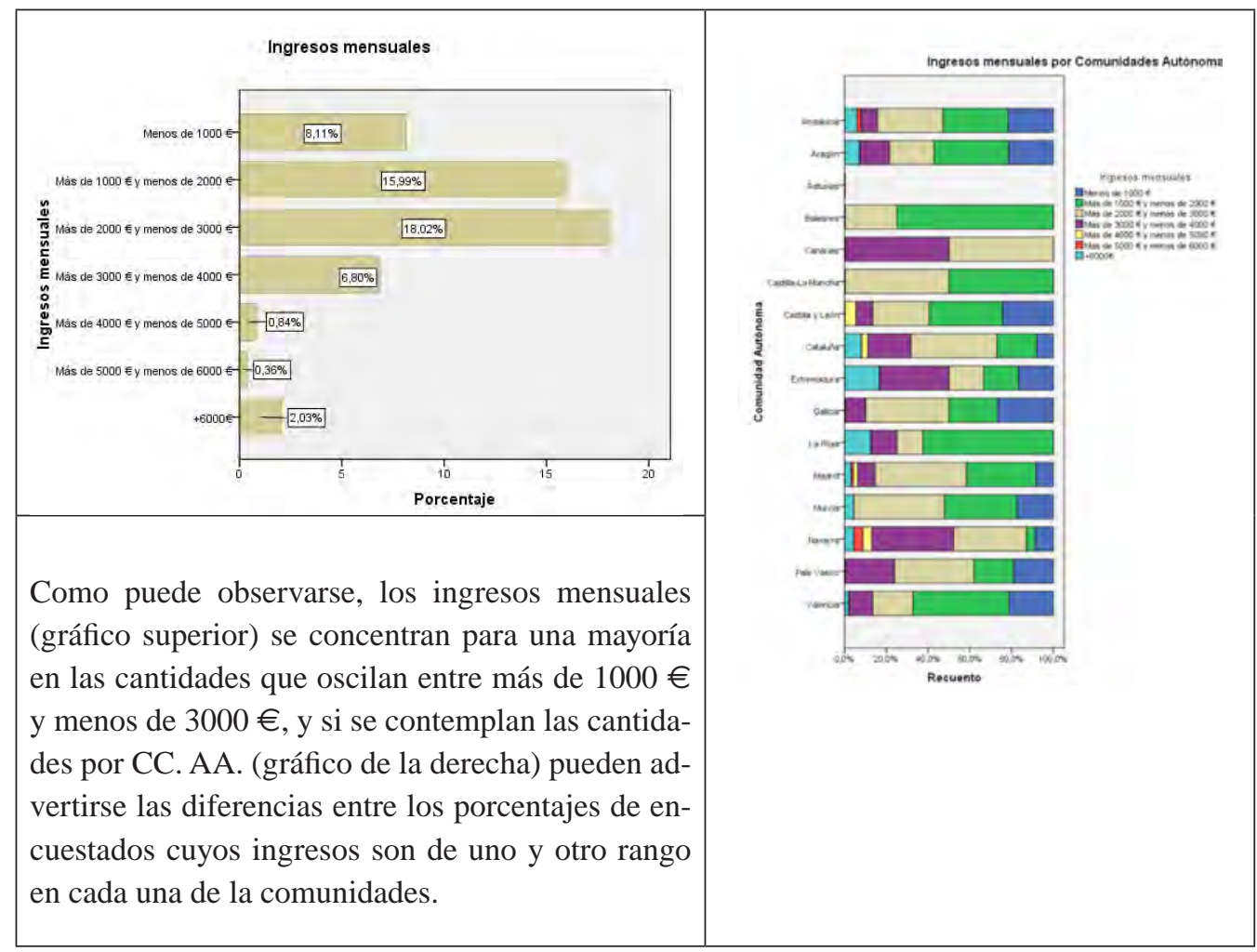


A la vista de las tablas anteriores, lo primero a destacar es que esta pregunta solo fue contestada por el $47,9 \%$ de los encuestados. Lo segundo, que el ingreso económico de la muestra de encuestados que responden es más bien modesto si se compara con otros países europeos, cuya media es muy superior, especialmente en Reino U nido, Suiza u Holanda (cfr. https://clionauta.wordpress.com/2008/05/15/salarios-del-profesorado-universitario/). Y lo tercero, que los intervalos de ingresos económicos se distribuyen de manera muy poco uniforme cuando se comparan por comunidades autónomas, lo cual muestra una desigual política científica en lo que atañe a la remuneración de los docentes investigadores.

\section{CONCLUSIONES}

Hasta aquí se han expuesto y comentado con mayor o menor detalle los perfiles de las respuestas de los investigadores censados en centros universitarios españoles con docencia reglada en grado y posgrado en Comunicación, invitados a cumplimentar un cuestionario que aspiraba a sondear las experiencias de su actividad investigadora reciente. Fueron invitados por correo electrónico a abrir el formulario en línea mediante un enlace y una contraseña 2418 doctores censados como docentes en aquellos centros universitarios, más un número indefinido de doctores sin docencia, miembros de sociedades científicas y doctorandos inscritos en programas de doctorado, a los que respectivamente cursaron la invitación los responsables de las sociedades científicas y los coordinadores de los programas de doctorado.

Respondieron total o parcialmente la encuesta 838 investigadores, que representaban un $34,66 \%$ del censo $y$, hecha la medida de la representatividad de esta muestra respecto al censo, se comprobó que lo era por los estratos de universidad y comunidad autónoma. A este número de investigadores del censo cuyas respuestas han sido tratadas y comentadas (838) se puede añadir como dato a destacar que contestaron la encuesta hasta el final 496, mientras que el resto (342) no la completó. Por otra parte, es también indicativo que, del universo de los 2418 que recibió la invitación, 416 la abrieron y la abandonaron acto seguido sin respuesta a ninguna pregunta.

El formulario comenzaba por preguntar sobre el conocimiento previo que el encuestado podía tener del Proyecto $\mathrm{M}$ apCom, y se comprobó que el interés mostrado al contestar la encuesta no se debió al conocimiento previo sobre este proyecto.

Los datos tratados y comentados sobre el perfil del investigador pueden resumirse así: 
- Entre los investigadores, la antigüedad como doctor que predomina abarca el segmento de 10 a 30 años y solo un $3 \%$ tiene antigüedad de más de 30 años. Por CC. A A ., Valencia, M adrid y A ndalucía están por encima del 14\% en la proporción de doctorandos que contestan la encuesta.

- M ás de la mitad de nuestros investigadores (un 51,1\%) carece de sexenios; el $15,5 \%$ tiene uno; el $11,3 \%$ solo tiene dos, y con tres sexenios o más solo hay un $6 \%$, y esto es así con independencia de la edad.

- Carece de la experiencia como IP un 44,4\%, y al menos una vez lo ha sido el $14,3 \%$, y de 2 a 5 veces, el $12,5 \%$.

- Un $33,9 \%$ dice que nunca ha dirigido una tesis doctoral y un $14,6 \%$ que nunca ha dirigido un TFM o un TFG.

- Cuatro de cada diez tienden a considerar bastante relevantes las valoraciones recibidas por evaluaciones de proyectos, artículos, tesis, etc., con independencia de que se trate de valoraciones aportadas en calidad de IP, de miembro de equipos de investigación o de directores académicos.

A sí pues, teniendo en cuenta estos datos, el perfil del universo representado por esta muestra indica una dedicación precaria a la actividad investigadora ya que, siendo la antigüedad dominante en el doctorado de 10 a 30 años, es revelador el pobre número de sexenios, de proyectos I+D y de dirección académica de tesis y de TFM, y es incongruente, sin embargo, que se aprecien como bastante rel evantes las evaluaciones recibidas. ¿Indica esto la carencia de expectativas?

L a adscripción profesional como investigador permite completar este perfil con datos tales como universidad donde presta sus servicios, rango académico/laboral, participación en grupos/equipos de investigación, comunidad autónoma, docencia ejercida, etc. Estos datos pueden resumirse así:

- Usa y consulta registros internacionales y nacionales de investigación más del $60 \%$ de los encuestados.

- Cinco de cada diez encuestados dejan sin contestar la pregunta sobre participación en sociedades científicas.

- Cuatro de cada diez encuestados son contratados, ya sea de forma indefinida $(20,8 \%)$ o temporal $(21,2 \%)$, mientras que los titulares y catedráticos, sean interinos o de plantilla, suman otros cuatro de cada diez, si bien los catedráticos apenas Ilegan al 6,3\%.

En resumen, el pobre perfil anterior en cuanto a dedicación a la actividad investigadora se completa al añadir aquí el dato de la desconexión de sociedades científicas y el 
relacionado con los rangos de la vinculación laboral cuyas precarias condiciones obstaculizan participar en investigaciones a medio y largo plazo, pues las convocatorias estatales de proyectos imponen como requisito que en los equipos de investigación deban figurar, mientras duran los proyectos, profesores con vinculación permanente (funcionarial o contractual), excluyendo así a investigadores con vinculación temporal. Y, en consecuencia, también resulta explicable la carencia o reducido número de sexenios reconocidos por la actividad investigadora.

Para recabar más información referida a la experiencia personal en la actividad investigadora desarrollada, hay que tomar en consideración la compatibilidad de la dedicación a la docencia con la dedicada a la investigación. En este sentido se manifiesta por parte de los encuestados que el reconocimiento académico de la dedicación docente va en detrimento de la actividad investigadora, aunque esta sea requerida para la promoción de la carrera universitaria. M ás aún, la docencia exclusiva en posgrado, que sería la más cercana a las exigencias de la actividad investigadora, es absolutamente minoritaria para todas las titulaciones, de forma que lo más frecuente es que si hay docencia en posgrado también sea compartida con el grado, presumiblemente perjudicando así la dedicación a la actividad investigadora.

Por otra parte, la evaluación de la calidad investigadora es valorada no tanto por la excelencia de sus resultados, por el avance de conocimiento y por retornos sociales como por el impacto de las publicaciones conforme al número de citas entre las élites de investigadores, mostrándose así más una competencia corporativa entre ellos que una competencia científica por el conocimiento y sus aplicaciones.

También por la integración en equipos de investigación se ventila el conflicto entre estas competencias corporativas y científicas. A sí, dice carecer de adscripción a proyectos vigentes un $15,8 \%$, y manifiesta su integración en ellos un $40,3 \%$. No obstante, los equipos y grupos de proyectos son efímeros, pues su duración suele ser la misma que la del proyecto, de tres años en su mayoría. En cambio, la participación en grupos de investigación consolidados (GIC) es declarada por una mayoría que roza el $55 \%$, siendo en calidad de IP un 9,2\%, y en calidad de miembros de los equipos de trabajo 0 del grupo de investigadores un $6,4 \%$ y un $41,5 \%$ respectivamente.

L as líneas de investigación, la fijación de objetivos, la elección de objetos de estudio y el tipo de técnicas empleadas para elaborar e interpretar datos es una información que permite, por otra parte, aproximarse a las representaciones que los propios investigadores tienen de su actividad, determinando con ello el producto de la investigación. Pues bien, a este propósito conviene resal tar estos datos obtenidos:

- A propósito de las líneas de investigación 479 de los 838 encuestados (el 57,2\%) brindaron palabras clave para describir líneas de investigación. El resultado pue- 
de resumirse en los siguientes campos de estudio: comunicación mediática, comunicación organizacional, comunicación de grupos, comunicación interpersonal, teoría y epistemología y métodos y técnicas. Es la comunicación mediática la que destaca sobre las demás como objeto de estudio. Hay dos razones para esta dominancia: desde su origen, nuestras facultades universitarias se orientaron a cumplir la función social de la formación superior de profesionales para los medios, sin orientarse a la formación de profesionales en otras prácticas sociales de la comunicación, como puede ser la educación, la sanidad, etc. y, hoy día, por otra parte, la emergencia de las prácticas mediadoras del universo digital vienen a sobrerrepresentar este perfil en la elección de campos de estudio.

- Dada la prioridad del objeto de estudio integrado en el campo de la comunicación mediática, no es de extrañar que las técnicas más utilizadas sean las de análisis documentales (análisis de contenido, análisis del discurso, análisis de documentación).

- Los encuestados comparten similar frecuencia en la elección de objetivos de investigación sobre objetos de estudio mayoritariamente del campo de la comunicación mediática, siendo el más frecuente de estos objetivos el de «describir» y el menos frecuente el de «intervenir», lo que es indicador de que asistimos a los primeros desarrollos de una disciplina más que al desarrollo característico de su madurez, lo que se traduciría en un mayor número de investigaciones centradas en aplicaciones sociales.

Estas apreciaciones que acaban de expresarse vienen confirmadas por las representaciones a propósito de la valoración de la experiencia investigadora en relación con el beneficio obtenido como retorno. En este sentido, en primer lugar, a las revistas se les atribuye en la val oración de retorno mayor influencia que a los libros y monografías y que a las actas de los congresos, confirmándose así una vez más que la competencia corporativa entre investigadores se impone sobre la competencia científica por el conocimiento y sus aplicaciones. A sí pues, aunque se constata una visión crítica sobre rendimientos de la investigación en lo que atañe a la publicación de resultados, se valoran menos las aplicaciones de estos que el marco financiero que los sustenta 0 , si se trata de las revistas de mayor impacto, que la elección de objetos y campos de estudio, especialmente.

Redundando en la valoración de las publicaciones científicas, estas vuelven a ser citadas como rendimiento mayoritariamente dominante en general, pero, sobre todo, para los objetivos de «explicar» y «evaluar»: función diagnóstica en detrimento de la función de intervención social. Se posterga incluso al retorno por divulgación/formación, que ocupa un tercer rango. 
En las trayectorias profesionales, la vida universitaria y la vida socioeconómica externa se dan la espalda. Se valoran como «nada importante» las contrataciones externas al ámbito universitario, así como, seguramente por despecho, las evaluaciones académicas referidas a los sexenios. La percepción entre los investigadores es que los rendimientos más valorados de la experiencia investigadora son las acreditaciones académicas, seguidas de la movilidad académica, lo cual confirma igual mente la competencia corporativa.

Como también forma parte de las representaciones de la propia actividad investigadora la valoración del esfuerzo que se invierte en ella, se preguntó sobre si este esfuerzo se producía aprovechando las condiciones positivas o si se invertía enfrentando condiciones negativas. Se preguntó así, en primer lugar, por la valoración positiva o negativa de las condiciones materiales: subvenciones y financiación, becas y contratos, trámites burocráticos e instalaciones y laboratorios; en segundo lugar, por la valoración positiva o negativa de las condiciones organizacionales: requisitos exigidos en convocatorias, programas de formación metodológica, redes de cooperación y conocimiento y recursos humanos en los equipos; y en tercer lugar, por la valoración positiva o negativa de las condiciones institucionales de los marcos en que se desarrollan los procesos de investigación: compatibilidad investigación y docencia, relaciones entre universidad y empresa, agencias de evaluación, calidad de congresos y participación, e indexación de revistas científicas. Pues bien, las condiciones materiales que acompañan los procesos de investigación son valoradas negativamente, salvo al referirse a instalaciones y laboratorios, cuya valoración es en conjunto positiva. En cambio, domina la valoración positiva a propósito de las condiciones organizacionales de los procesos de investigación, si bien los requisitos exigidos en convocatorias públicas y los programas de formación metodológica son valorados más negativamente que las redes de cooperación y conocimiento y los recursos humanos en los equipos. Finalmente, al apreciar las condiciones de los marcos institucionales, aparecen mayoritariamente valoraciones positivas solo al juzgar la calidad de congresos y participación, mientras que son más frecuentes las valoraciones extremadamente negativas (-3) al juzgar la compatibilidad entre investigación y docencia, las relaciones entre universidad y empresa y al juzgar las agencias de evaluación ANECA, ANEP, y CNEAI.

Por último, se persiguió conocer los rasgos referentes a los perfiles sociodemográficos del censo de investigadores en comunicación respondiendo a las preguntas sobre edad, género, formación de origen y rango de ingresos económicos por la actividad profesional. Y los rasgos más destacables son estos:

- Los perfiles sociodemográficos del censo de investigadores en comunicación responden a intervalos de edad y género que reúnen a más del $50 \%$ de investi- 
gadores mayores de 41 años y menores de 60. U na cuarta parte son menores de 40 años, y entre los séniores o mayores de 61, apenas uno de cada diez. Por lo que respecta al género, a medida que aumenta el rango de la edad disminuye la proporción de mujeres, y cuanto menor es la edad, mayor es el número de estas. Hay pues un techo de cristal a partir de los 40 años.

- Según la apreciación personal sobre la propia formación previa, son las humanidades y las ciencias sociales las más destacadas en su valoración, tanto si la referencia es a la formación preuniversitaria como a la formación de grado y posgrado. De forma inversa, las ciencias de la naturaleza y las matemáticas y tecnologías son formaciones previas apreciadas como satisfactorias cuando se refieren al nivel preuniversitario, mientras que casi dos terceras partes de los encuestados las consideran no procedentes referidas a la formación de grado y de posgrado. Con tales perfiles generales en la apreciación de la formación adquirida, no es de extrañar la dificultad que se impone a una política científica para transformar los diseños curriculares en beneficio de las ciencias experimentales, así como el esfuerzo añadido al que se enfrentan los académicos del ámbito de la comunicación para tener éxito en las evaluaciones a que someten sus trabajos para ser publicados en revistas cuyo impacto viene medido por parámetros habituales en el ámbito de las ciencias experimentales. Estas circunstancias vienen también a entorpecer las aspiraciones de los académicos de la comunicación para hacerse reconocer sus especial idades en los listados actual es de los códigos UNESCO y códigos NABS.

- En la mayor parte de los casos, los ingresos de los investigadores oscilan entre más de $1000 €$ y menos de $3000 €$, y, si se contemplan las cantidades por CC. A A ., resalta la ausencia de uniformidad. Por supuesto, las diferencias en la distribución y cuantía de las cantidades con que se remunera a los académicos son atribuibles a la política científica que estratégicamente se establece en cada una de la comunidades.

En conclusión, con esta encuesta, que es la primera vez que se aplica a un censo de investigadores tan amplio en un país y de una especialidad tan concreta como esta, hemos podido aproximarnos a sus perfiles de encuadre en su actividad investigadora y a sus representaciones sobre la propia experiencia y actividad en curso, gracias a lo cual se ha podido descubrir el capital humano que lo integra y sus fortalezas y debilidades al objeto de poder desarrollar las políticas científicas más adecuadas a su potencial. 
BIBLIOGRAFÍA

Alsina, M iguel-Rodrigo; García, Leonarda (2010). «Communication theory and research in Spain: A paradigmatic case of a socio-humanistic discipline». European Journal of Communication, 25(3), 273-286. doi: https://dx.doi. org/10.1177/0267323110373458

A rcila, Carlos; Piñuel, José-L uis; Calderín, M abel (2013). La e-investigación de la Comunicación: actitudes, herramientas y prácticas en investigadores iberoamericanos. [The e-Research on M edia \& Communications: A ttitudes, Tools and Practices in Latin America Researchers]. Comunicar 40, 111-118. doi:http://dx.doi. org/10.3916/C 40-2013-03-01

A sociación para la Investigación de M edios de Comunicación, A IM C (2016): M arco general de los medios en España, 2015, http://www.aimc.es/-Descarga-M arco-General-A sociados-.html .

B achelard, Gastón (1973). Epistemología. Barcelona: A nagrama.

B lázquez, M anuel (2015): Tesis D octorales en las U niversidades E spañolas durante el periodo 1977-2014. En: http://mblazquez.es/tesis-doctorales-en-las-universidades-espanolas-durante-el-periodo-1977-2014/.

Cáceres, M aría-D olores; Caffarel, C armen (1993a). «La investigación sobre comunicación en España. Un balance cualitativo». Telos, 32, 109-124. En: https://tel os.fundaciontelefonica.com/telos/anteriores/indices/28_37/autores/autores_c.htm

Caffarel, Carmen, Izquierdo, Patricia y Núñez Sonia (2017). ¿Cómo investiga la mujer cuando investiga sobre la mujer en comunicación? En Tecnos (en impresión).

Caffarel, Carmen; Cáceres, M aría-D olores (1993b). La comunicación en España: planteamientos temáticos y metodológicos entre 1987 y 1990. La investigación en España. M onografías C.I.N.C.O, Cuadernos de Investigación en Comunicación 29, 2330.

Caffarel, Carmen; Domínguez, M ilagros; Romano, Vicente (1989). El estado de la investigación en Comunicación en España (1978-1987), Cuadernos de investigación en Comunicación (C.I.N.C.O.) 3, 45-57.

Castillo, A ntonio; Xifra, Jordi (2006). Investigación bibliométrica de las tesis doctorales españolas sobre relaciones públicas (1965-2005). A nàlisi 34, 141-161.

Deacon, Terence W. (1997). The symbolic species: the co-evolution of language of the brain. Nueva York, W. W. Norton \& Co.

Delgado, Emilio; Torres, Daniel, Contreras, Evaristo et al. (2006). «A nálisis bibliométrico y de redes sociales aplicado a las tesis bibliométricas defendidas en España (1976-2002): temas, escuelas científicas y redes académicas». Revista Española de 
Documentación Científica, 29(4), 493- 524. doi: https://dx.doi.org/10.3989/ redc.2006.v29.i4.306

Delicado, A na (2011). «¿Para qué sirven las sociedades científicas?», en Revista Iberoamericana de Ciencia, Tecnología y Sociedad - CTS, septiembre 2011.

EC 3M etrics (2015). A nálisis de los resultados de la Convocatoria de Proyectos I+D +I del Plan Nacional de Investigación. En: https://goo.gl/pt0IX S.

Fernández, David; M asip, Pere (2013). Tres décadas de investigación española en comunicación: hacia la mayoría de edad. [Three Decades of Spanish Communication Research: Towards L egal A ge] Comunicar, 41, 15-24. doi: http://dx.doi.org/10.3916/ C41-2013-01

Fuentes, Eulàlia; A rguimbau, Llorenç (2010). «L as tesis doctorales en España (19972008): análisis, estadísticas y repositorios cooperativos». Revista Española de Documentación Científica, 33(I), 63-89. doi: http://dx.doi.org/10.3989/redc.2010.1.711 Gama Cubas, M arina (2017). «La universidad española lejos de la paridad en las cátedras», en El M undo, 27/09/2017. disponible en http://www.elmundo.es/grafico/sociedad/2017/09/27/59b8e122268e3eae4f8b4672.html

Garfinkel, Harold (1967). Studies in Ethnomethodology. Nueva York: Prentice-Hall.

I gartua, J uan J osé (2017). «Cuestiones de calidad y metodología en la investigación en Comunicación en España», en Bernardo Díaz N osty Ruth de Frutos (coords.), Tendencias de la investigación universitaria española en Comunicación, $N$ avarra: Thomson Reuters, A ranzadi. pp. 269-286.

Jones, Daniel E. (1994). «Investigació sobre comunicació al'Espanya dels noranta». Centre d'Investigació de la Comunicació i U niversitatA utònoma de B arcelona. Cultura y comunicación social: América Latina y Europa Ibérica. Barcelona: Generalitat de Catalunya.

Jones, Daniel E. (1998). Investigación sobre comunicación en España. Evolución y perspectivas. Zer, 5, 13-51.

Jones, Daniel E.; Baró, Jaume, L anda, Carles et al. (2000). Investigación sobre comunicación en España: Aproximación bibliométrica a las tesis doctorales (1926-1998). B arcelona: ComC at.

Leydesdorff, Loet. (2003). A Sociological Theory of Communication. The Self-O rganization of the Knowledge-Based Society. Parkland, FL: U niversal Publishers.

Lledó, Emilio (1961). El silencio de la escritura, M adrid: Espasa

López, Pablo; Vicente, M iguel. (2011): «M étodos y técnicas de investigación dominantes en las revistas científicas españolas sobre comunicación (2000-2009)». En J 0sé-L uis Piñuel, Carlos L ozano y A lberto García (Eds.): Investigar la comunicación en España, (pp. 665-679). Madrid: A E-IC/URJC. 
Lozano, Carlos; Gaitán, Juan-A ntonio (2011). «Dedicación a la investigación y a la docencia universitaria en comunicación: compatibilidad y perfiles en España, Europa y A mérica Latina». En Piñuel, José-Luis,; Lozano, Carlos y A lberto García (Eds.). Investigar la Comunicación en España (pp.587-595). Facultad de Ciencias de la Comunicación. Fuenlabrada: U niversidad Rey J uan Carlos.

L uhmann, Niklas (1991). Sistemas sociales. Lineamientos para una teoría general. M éxico: Universidad Iberoamericana.

M artínez, M . (2009). «L a investigación sobre comunicación en España. Evolución histórica y retos actual s». [The Communication Research in Spain. Historical evolution and current challenges]. Revista Latina de Comunicación Social, 64, 1-14. doi: http://dx.doi.org/10.4185/R LCS-64-2009-800-01-14

M artínez, M anuel; Saperas, Enric (2011). «La investigación sobre comunicación en España (1998-2007)». A nálisis de los artículos publicados en revistas científicas, Revista Latina de Comunicación Social, 66, 101-129. doi: http://dx.doi.org/10.4185/ RLCS-66-2011-926-101-129

M aturana, H umberto y Varela, Fernando (1973). D e máquinas y seres vivos. Santiago de Chile: Ed. Universitaria;

- (1996). El árbol del conocimiento. M adrid: Debate [1984]

MECD (2017). Estadísticas e informes universitarios. Disponible en www.mecd.gob. es/servicios-al-ciudadanomecd/estadisticas/educacion.html.

Piñuel, José Luis (2009). La comunicación como objeto científico de estudio, como campo de análisis y como disciplina científica, Contratexto Digital, 18, 1-22.

Piñuel, J osé L uis y L ozano, Carlos (2006). Ensayo general sobre la comunicación. Barcelona: Paidos.

Piñuel, J osé-Luis, (2011) «La docencia y la investigación universitarias en torno a la Comunicación como objeto de estudio en Europa y A mérica L atina». [Teaching and Research on Communication as an object of study in European and L atin-A merican universities]. Cuadernos A rtesanos de Latina. N. 15. La L aguna-Tenerife: SLCS.

Piñuel, J osé-L uis; L ozano, Carlos; Gaitan, Juan-A ntonio. (2016). «Propuesta de estudio para realizar un mapa de la investigación en comunicación en A mérica L atina», Revista Latinoamericana de Ciencias de la Comunicación, vol. 11, n.을 22 (12), 45-53. Piñuel, José-Luis; Lozano, Carlos; García, Alberto (2011). «Investigar la comunicación en España». Libro deA ctas del I Simposio Nacional de Grupos consolidados de Investigación en Comunicación y del I Congreso nacional de M etodología de la I nvestigación en Comunicación. Facultad de Ciencias de la Comunicación. Fuenlabrada-M adrid: A EIC/URJC. Piñuel, J osé-L uis; Sánchez de Diego, M anuel (2015). En busca del arca perdida: solicitud de información de proyectos de investigación sobre comunicación. En Rodrigo 
Cetina et al. (Eds.). Media and Journalists in the Age of O pen Government and Transparency. M adrid: UCM .

Ranke, L eopold (1954). E pochen. Darmstadt.

Repiso, Rafael; D elgado, E milio; Torres, Daniel (2011a). «A nálisis bibliométrico de la producción española de tesis doctorales sobre Cine. 1978- 2007». En Ivan Bort, Shaila García y M arta M artín (Eds.), A ctas del IV Congreso Internacional sobre A nálisis Fílmico, nuevas tendencias e hibridaciones de los discursos audiovisuales en la cultura digital contemporánea, (pp. 976- 987). M adrid: Ediciones de la Ciencias Sociales de M adrid. doi: http://dx.doi.org/10.6035/978-84-87510-57-1.2011.80 Repiso, Rafael; Delgado, Emilio; Torres, Daniel (2011b). Análisis bibliométrico y de redes sociales en tesis doctorales españolas sobre televisión (1976/2007) [Bibliometric and Social N etwork A nalysis A pplied to Television Dissertations Presented in Spain (1976/2007)]. Comunicar, 37(X IX ), 151-159. doi: http://dx.doi.org/10.3916/ C37-2011-03-07.

Saperas, Enric (2016). «Cuatro décadas de investigación comunicativa en España. Los procesos de institucionalización y de profesionalización de la investigación (19712015)» en: A nuario Electrónico de Estudios en Comunicación Social Disertaciones. 9 (2). pp. 27-45.

SEO Economic Research (2007). International wage differences in academic occupations. Disponible en https://clionauta.wordpress.com/2008/05/15/salarios-del-profesorado-universitario/

Tuñez López, Miguel (2014). Perfiles de Comunicación en Google Scholar Metrics, índice h y nuevas estrategias de difusión de la investigación en $\mathrm{H}$ istoria y Comunicación Social, vol. 19, n.e esp. marzo, pp. 15-26.

U M yC Unidad M ujer y Ciencia (2016). Informe Científicas en cifras 2015, M INECO. Disponible en http://www.idi.mineco.gob.es/stfls/M ICINN/M inisterio/FICHEROS/ Informe_Cientificas_en_Cifras_2015_con_A nexo.pdf

Vicente, M iguel; Gonzáles, Tecla; Pacheco, M arta (Coord.), (2013). «Investigar la comunicación hoy: revisión de políticas científicas y aportaciones metodológicas». A ctas del II Congreso Nacional sobre M etodología de la Investigación en Comunicación y del Simposio Internacional sobre Política Científica en Comunicación. Segovia: A EIC-UVA.

Vigotsky, L.S. (1988). El desarrollo de los procesos psicológicos superiores, Crítica, Grijalbo, M éxico.

X ifra, J ordi; Castillo, A ntonio (2006). Forty years of doctoral public relations research in Spain: A quantitative study of dissertation contribution to theory development. Public relations Review, 32(3), 302-308. 